\title{
Robustness to Axon Initial Segment Variation Is Explained by Somatodendritic Excitability in Rat Substantia Nigra Dopaminergic Neurons
}

\author{
ĐEstelle Moubarak, ${ }^{1}$ @Dominique Engel, ${ }^{2}$ Martial A. Dufour, ${ }^{1}{ }^{\oplus}$ Mónica Tapia, ${ }^{1}$ @Fabien Tell, ${ }^{1 *}$ \\ and ๑Jean-Marc Goaillard ${ }^{1 \star}$ \\ 1Unité Mixte de Recherche_S 1072, Aix Marseille Université, Institut National de la Santé et de la Recherche Médicale, Faculté de Médecine Secteur Nord, \\ 13015 Marseille, France, and 2Laboratory of Neurophysiology, GIGA-Neurosciences, Université de Liège, 4000 Liège 1, Belgium
}

In many neuronal types, axon initial segment (AIS) geometry critically influences neuronal excitability. Interestingly, the axon of rat SNc dopaminergic (DA) neurons displays a highly variable location and most often arises from an axon-bearing dendrite (ABD). We combined current-clamp somatic and dendritic recordings, outside-out recordings of dendritic sodium and potassium currents, morphological reconstructions and multicompartment modeling on male and female rat SNc DA neurons to determine cell-to-cell variations in AIS and ABD geometry, and their influence on neuronal output (spontaneous pacemaking frequency, action potential [AP] shape). Both AIS and ABD geometries were found to be highly variable from neuron to neuron. Surprisingly, we found that AP shape and pacemaking frequency were independent of AIS geometry. Modeling realistic morphological and biophysical variations helped us clarify this result: in SNc DA neurons, the complexity of the ABD combined with its excitability predominantly define pacemaking frequency and AP shape, such that large variations in AIS geometry negligibly affect neuronal output and are tolerated.

Key words: action potential; axon initial segment; modeling; robustness; sodium channels; variability

Significance Statement

In many neuronal types, axon initial segment (AIS) geometry critically influences neuronal excitability. In the current study, we describe large cell-to-cell variations in AIS length or distance from the soma in rat substantia nigra pars compacta dopaminergic neurons. Using neuronal reconstruction and electrophysiological recordings, we show that this morphological variability does not seem to affect their electrophysiological output, as neither action potential properties nor pacemaking frequency is correlated with AIS morphology. Realistic multicompartment modeling suggests that this robustness to AIS variation is mainly explained by the complexity and excitability of the somatodendritic compartment.

\section{Introduction}

In mammalian neurons, it is currently accepted that the action potential (AP) is initiated in the axon initial segment (AIS), a subcellular compartment comprising a particularly high density

\footnotetext{
Received Oct. 29, 2018; revised March 27, 2019; accepted March 27, 2019.

Author contributions: E.M., D.E., M.A.D., M.T., and F.T. performed research; E.M., D.E., M.A.D., M.T., F.T., and J.-M.G. analyzed data; E.M., D.E., F.T., and J.-M.G. wrote the paper; F.T. and J.-M.G. designed research.

This work was supported by the French Ministry of Research doctoral fellowship to E.M., French National Research Agency ANR JCJC Grant ROBUSTEX to J.-M.G., Fondation France Parkinson doctoral fellowship to M.A.D., European Research Council (Consolidator Grant 616827 CanaloHmics to J.-M.G., supporting M.T.), and the Belgian FRS-FNRS U.N002.13 and T.N0015.13 to D.E. We thank Dr. Michael Seagar for helpful comments on the manuscript; Dr. Vincent Seutin for helpful discussions on the subject; and the GIGA-Imaging (Liège) and the CMI imaging (Marseille) facilities for help with the acquisition of confocal images.

The authors declare no competing financial interests.

*F.T. and J.-M.G. contributed equally to this work.

Correspondence should be addressed to Jean-Marc Goaillard at jean-marc.goaillard@univ-amu.fr.

https://doi.org/10.1523/JNEUROSCI.2781-18.2019

Copyright $\odot 2019$ the authors
}

of voltage-gated sodium channels (Coombs et al., 1957; Kole et al., 2008; Debanne et al., 2011; Kole and Stuart, 2012), due to the presence of the AIS-specific anchoring protein ankyrinG (Rasband, 2010). Some neurons even express different subtypes of sodium channels in the proximal and distal parts of the AIS (Nav1.1/Nav1.2 and Nav1.6, respectively) (Van Wart et al., 2007; Lorincz and Nusser, 2008; Hu et al., 2009), and experimental and theoretical studies demonstrated that the AP can be initiated specifically in the distal (Palmer and Stuart, 2006; Kole et al., 2008; $\mathrm{Hu}$ et al., 2009) or the proximal part of the AIS (Meeks and Mennerick, 2007). Consistent with the specific localization of the channels responsible for AP initiation, it has been demonstrated that changes in AIS geometry (length or distance from soma) can modulate neuronal excitability, firing frequency or AP properties in several neuronal types (Kuba et al., 2006, 2014; Grubb and Burrone, 2010; Wefelmeyer et al., 2015; Lezmy et al., 2017). 
However, these links between AIS geometry and neuronal excitability have been determined on neuronal types that are driven by dense temporally and spatially ordered synaptic inputs. The question arises then whether the constraints on AIS geometry are similar in spontaneously active neuronal types, where the main driving force for activity comes from the intrinsic conductances expressed by the neuron, and not from synaptic inputs. The midbrain dopaminergic (DA) neurons of the SNc are spontaneously active in the absence of synaptic inputs (Grace and Onn, 1989), generating a regular tonic (also known as pacemaker) activity relying on many distinct voltage- and calcium-gated ion channels (Liss and Roeper, 2008; Gantz et al., 2018). These neurons are also known to project a long and highly ramified axon to the dorsal striatum where they release dopamine (Prensa and Parent, 2001; Matsuda et al., 2009). Interestingly, while in most mammalian neurons the axon emerges from the soma (Kole and Brette, 2018), the axon of DA neurons often arises from a primary or secondary dendrite, at distances from the soma as large as $200 \mu \mathrm{m}$ in the adult rat (Grace and Bunney, 1983; Häusser et al., 1995; Gentet and Williams, 2007; Blythe et al., 2009; Meza et al., 2018). Despite these surprisingly large cell-to-cell variations in axon location (and thus potentially of the AIS), only one recent study performed in mouse SNc neurons was dedicated to studying the link between AIS geometry and excitability in these cells (Meza et al., 2018).

We investigated the link between AIS geometry and excitability (spontaneous firing and AP shape) using a combination of current-clamp and voltage-clamp electrophysiological recordings, morphological reconstructions, and computational modeling on rat SNc DA neurons. In contrast with the previously cited studies (Kuba et al., 2006; Grubb and Burrone, 2010; Meza et al., 2018), cell-to-cell variations in AIS geometry did not seem to significantly influence the excitability of DA neurons, as neither the level of spontaneous firing nor AP properties showed correlations with AIS length or soma-AIS distance. Dendritic voltageclamp recordings confirmed the presence of a significant density of sodium channels in the dendrites, and neuronal reconstructions revealed an unexpected morphological complexity of the axon-bearing dendrite. Multicompartment computational modeling then suggested that the presence of somatodendritic sodium channels associated with the morphological complexity of the dendritic compartment is sufficient to explain the lack of dependence between AIS geometry and excitability in these neurons. Our results suggest that the peculiar morphological and biophysical profile of the dendritic arborization in these neurons minimizes the influence of the AIS on excitability, such that its precise geometry ceases to represent a significant constraint for this cell type.

\section{Materials and Methods}

Acute midbrain slice preparation. Acute slices were prepared from P16P22 (mean $=19, n=141)$ Wistar rats of either sex. All experiments were performed according to the European (Council Directive 86/609/EEC) and institutional guidelines for the care and use of laboratory animals (French National Research Council; protocol 1210, Commission d'Ethique Animale, University of Liège; Comité déontologique, Belgium). Rats were anesthetized with isoflurane (CSP) in an oxygenated chamber (TEM SEGA) and decapitated. The brain was immersed briefly in oxygenated ice-cold low-calcium aCSF containing the following (in mм): $125 \mathrm{NaCl}, 25 \mathrm{NaHCO}_{3}, 2.5 \mathrm{KCl}, 1.25 \mathrm{NaH}_{2} \mathrm{PO}_{4}, 0.5 \mathrm{CaCl}_{2}, 4$ $\mathrm{MgCl}_{2}, 25$ D-glucose; or $87 \mathrm{NaCl}, 25 \mathrm{NaHCO}_{3}, 2.5 \mathrm{KCl}, 1.25 \mathrm{NaH}_{2} \mathrm{PO}_{4}$, $0.5 \mathrm{CaCl}_{2}, 7 \mathrm{MgCl}_{2}, 10$ D-glucose, 75 sucrose; $\mathrm{pH} 7.4$, oxygenated with $95 \% \mathrm{O}_{2} / 5 \% \mathrm{CO}_{2}$ gas. The cortices were removed and then coronal midbrain slices $(250$ or $300 \mu \mathrm{m})$ were cut on a vibratome (VT 1200 or 1200S,
Leica Microsystems) in oxygenated ice-cold low-calcium aCSF. Following 20-30 min incubation in $32^{\circ} \mathrm{C}$ oxygenated low calcium aCSF, the slices were incubated for at least $30 \mathrm{~min}$ in oxygenated aCSF $(125 \mathrm{NaCl}$, $25 \mathrm{NaHCO}_{3}, 2.5 \mathrm{KCl}, 1.25 \mathrm{NaH}_{2} \mathrm{PO}_{4}, 2 \mathrm{CaCl}_{2}, 2 \mathrm{MgCl}_{2}$, and 25 glucose, $\mathrm{pH} 7.4$, oxygenated with $95 \% \mathrm{O}_{2} 5 \% \mathrm{CO}_{2}$ gas) at room temperature before electrophysiological recordings.

Drugs. For current-clamp recordings, picrotoxin (100 $\mu \mathrm{M}$, SigmaAldrich) and kynurenate (2 mM, Sigma-Aldrich) were bath-applied via continuous perfusion in aCSF to block inhibitory and excitatory synaptic activity, respectively. TTX citrate ( $1 \mu \mathrm{M}$, Tocris Bioscience or Abcam) was bath-applied to verify the TTX sensitivity of voltage-gated sodium currents and to isolate potassium currents. In addition, cadmium chloride (200 $\mu \mathrm{M}$, Sigma-Aldrich) was bath applied to block voltage-gated calcium channels during potassium current recordings.

Electrophysiology recordings and analysis. All recordings (141 cells from 64 rats) were performed on midbrain slices continuously superfused with oxygenated aCSF. Picrotoxin and kynurenate were systematically present for all recordings to prevent contamination of the intrinsic activity by spontaneous glutamatergic and GABAergic synaptic activity. Patch pipettes $(1.8-4 \mathrm{mOhm}$ for somatic recordings, $5-13 \mathrm{mOhm}$ for dendritic or outside-out recordings) were pulled from borosilicate glass (GC150TF-10, Harvard Apparatus) on a DMZ Universal Puller (Zeitz Instruments) or a P-97 horizontal puller (Sutter Instruments). For most recordings, patch solutions contained the following (in $\mathrm{mM}$ ): $20 \mathrm{KCl}, 10$ HEPES, 10 EGTA, $2 \mathrm{MgCl}_{2}$, $2 \mathrm{Na}-\mathrm{ATP}$, and $120 \mathrm{~K}$-gluconate, pH 7.4, 290-300 mOsm. A different solution composed of the following (in mM): 120 Cs gluconate, $20 \mathrm{CsCl}, 2 \mathrm{MgCl}_{2}, 2 \mathrm{Na}_{2} \mathrm{ATP}, 10 \mathrm{HEPES}$, and 10 EGTA, pH 7.3, 300 mOsm was used to record $\mathrm{Na}^{+}$currents in outsideout patches. Neurobiotin $(0.05 \%$; Vector Labs) or biocytin $(0.1 \%-0.2 \%$, Invitrogen) was included in the intracellular solution to allow morphological reconstruction and identification of dopaminergic neurons using post hoc TH immunolabeling (Amendola et al., 2012). Whole-cell recordings were made from SNc dopaminergic neurons visualized using infrared differential interference contrast videomicroscopy (QImaging Retiga camera; Olympus BX51WI microscope or Newvicon tube in NC-70 Dage-MTI, Carl Zeiss FS microscope) and identified as previously described (Amendola et al., 2012), subsequently dendrites were visualized with a $2 \times-4 \times$ changer (Dodt, Luigs Neumann). Whole-cell currentclamp recordings with a series resistance $<10 \mathrm{mOhm}$ (soma) or $<25$ $\mathrm{mOhm}$ (dendrites) were included in the study. Capacitive currents and liquid junction potential $(13.2 \mathrm{mV})$ were compensated online, and offset potentials were measured after removing the pipette from the neuron. Bridge balance $(100 \%, 10 \mu \mathrm{s})$ was used in current-clamp to compensate series resistance. For voltage-clamp recordings, a series resistance compensation of $60 \%$ (10 $\mu$ s) was used. Recordings with offset values $>1 \mathrm{mV}$ were discarded from the analysis. Recordings were acquired at $50 \mathrm{kHz}$ and were filtered with a low-pass filter (Bessel characteristic between 2.9 and $5 \mathrm{kHz}$ cutoff frequency).

The interspike interval (ISI) and ISI coefficient of variation $\left(\mathrm{CV}_{\mathrm{ISI}}\right)$ were calculated from a minimum of $40 \mathrm{~s}$ of stable current-clamp recording (with no injected current) within the first 5 min of obtaining the whole-cell configuration. APs generated during this period of spontaneous activity were then averaged and the AP threshold, AP amplitude, and the duration of the AP at half of the maximal height of the AP (AP half-width) were measured. AP threshold was measured on the $\mathrm{d}^{2} \mathrm{v} / \mathrm{dt}^{2}$ versus $\mathrm{V}$ phase plane plot. To record voltage-gated $\mathrm{Na}^{+}$current in outside-out patches, a pulse sequence comprised of a $100 \mathrm{~ms}$ prepulse to $-120 \mathrm{mV}$, and a $30 \mathrm{~ms}$ test pulse to $0 \mathrm{mV}$ was generated. The holding potential before and after the pulse sequence was $-90 \mathrm{mV}$. For the recordings of voltage-gated $\mathrm{K}^{+}$current in outside-out patches, a pulse sequence comprised of a $150 \mathrm{~ms}$ prepulse to $-40 \mathrm{mV}$ followed by a 100 $\mathrm{ms}$ test pulse at $40 \mathrm{mV}$ was applied. The potential was briefly brought to $-40 \mathrm{mV}$ for $10 \mathrm{~ms}$ before returning to the holding potential of $-80 \mathrm{mV}$. For all outside-out recordings, voltage protocols were applied to outsideout patches once every $10 \mathrm{~s}$. Leak and capacitive currents were subtracted online using a $\mathrm{P}$ over -4 or $\mathrm{P}$ over -8 correction procedure. Current recordings are averages of $2-100$ sweeps. The patch area was estimated using the following equation: $a=12.6\left(1 / R_{\mathrm{p}}+0.018\right)$, where $a$ is the area $\left(\mu \mathrm{m}^{2}\right)$ and $R_{\mathrm{p}}$ is pipette resistance (mOhm) (Sakmann and Neher, 
Table 1. Equations governing the voltage dependence and kinetics of currents in the model

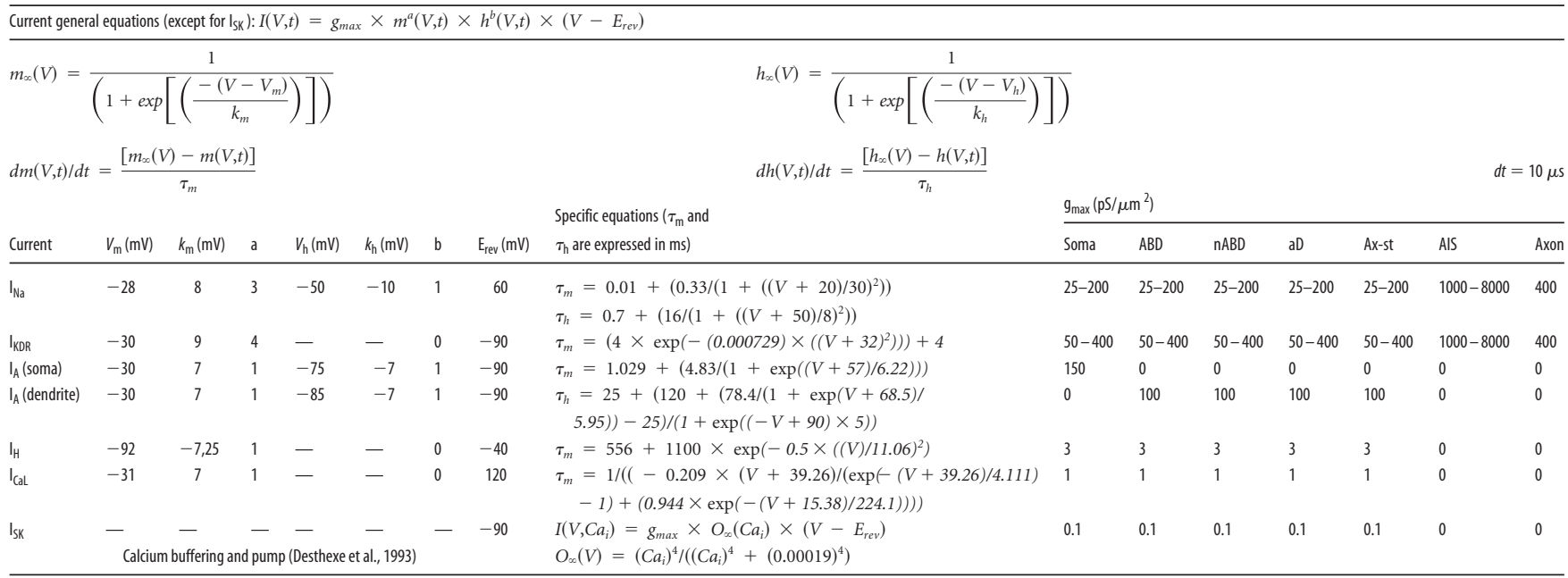

1995). Outside-out recordings were obtained from 29 neurons for sodium currents and 25 neurons for potassium currents. For sodium currents, multiple outside-out recordings (one somatic, one dendritic) were obtained from 2 neurons. After the outside-out recordings, a somatic whole-cell patch-clamp recording was performed to inject the neurobiotin necessary for post hoc reconstruction, which could be achieved in 20 neurons (22 outside-out recordings) and 14 neurons (14 outside-out recordings) for sodium and potassium currents, respectively. The range, average, and SD of sodium and potassium conductance values were calculated from the whole sample of recordings $(n=29 ; n=25)$, while only the measurements with post hoc reconstruction $(n=22 ; n=14)$ were used to analyze the somatodendritic distribution of conductance values.

Electrophysiology data acquisition and analysis. Data were acquired with an EPC 10/USB patch-clamp amplifier (HEKA Elektronik) and patchmaster software (HEKA Elektronik) or an Axopatch 200B amplifiers (Molecular Devices) connected to a PC via a Digidata 1440A interface (Molecular Devices) and pClamp 10.4 software (Molecular Devices). Analysis was conducted using FitMaster version 2x30 (HEKA Elektronik), Mathematica 9 (Wolfram Research), Stimfit 0.13 (Christoph Schmidt-Hieber, Institut Pasteur, Paris), Excel (Microsoft), and Anaconda/Spyder (Python 3.4).

Immunohistochemistry and morphological reconstruction. Acute slices containing Neurobiotin or biocytin tracer-filled cells were fixed $30 \mathrm{~min}$ in $4 \%$ PFA at $4^{\circ} \mathrm{C}$ and immunolabeled with anti-TH (chicken polyclonal, Abcam, 1:1000), anti-Ankyring (mouse monoclonal IgG2b, NeuroMab, 1:250 or mouse monoclonal, Invitrogen, 1:100), goat anti-mouse IgG2b AlexaFluor-488 (Invitrogen; 1:1000 $2 \mu \mathrm{g} / \mathrm{ml}$ ), and goat anti-chicken AlexaFluor-633 (Invitrogen; 1:3000; $1.66 \mathrm{ng} /$ $\mathrm{ml}$ ) or donkey anti-mouse AlexaFluor-647 (Invitrogen; 1:250). Streptavidin AlexaFluor-594 (Invitrogen; 1:12,000; $1.66 \mathrm{ng} / \mathrm{ml}$ ) or Fluorescein Avidin DCS (Lab Consult; $1 \mu \mathrm{l} / \mathrm{ml}$ ) were used to reveal neurobiotin and biocytin labeling, respectively. Slices were mounted in ProLong Antifade (Invitrogen) or Faramount mounting medium (Dako). Immunolabeling was viewed on an LSM 780 (Carl Zeiss) or an FV1000 (Olympus) confocal microscope, and images were captured using ZEN software (Carl Zeiss). Images were analyzed with Fiji/ImageJ software (Schindelin et al., 2012; Schneider et al., 2012; Rueden et al., 2017) and in particular neurons were reconstructed using the Simple Neurite Tracer plugin (Longair et al., 2011). Dendrite lengths were extracted directly from the paths traced through the stack images of the neurons. Soma volumes were estimated by using the "fill out path" method on Simple Neurite Tracer. For the diameters of the primary dendrites, auxiliary dendrites (aDs), axon, and AIS, the fluorescence histograms of branch sections were obtained and fitted with a Gaussian curve model. Diameters were then estimated as $3 \times$ SD of the Gaussian distribution.

Multicompartment modeling. Simulations were performed using NEURON 7.5 software (Carnevale and Hines, 2006). Neuronal mop- hologies included a simplified dopaminergic neuron based on average measurements and the realistic morphologies from the 37 fully reconstructed dopaminergic neurons. The simplified model consists of a cylindrical soma $(20 \times 20)$ attached to three non-axon-bearing $(n A B D)$ tapering dendrites ( $500 \mu \mathrm{m}$ long, tapering from 3 to $0.5 \mu \mathrm{m}$ ), an axonbearing dendrite $(\mathrm{ABD})$ dendrite $(x \times y)$, three aDs $(500 \mu \mathrm{m}$ long, tapering from 2 to $0.5 \mu \mathrm{m}$ ) and an axon subdivided into three compartments (axonstart, AIS, axon). Details of the morphology of the average model are provided in Figure 7.

For each compartment, membrane voltage was obtained as the time integral of a first-order differential equation as follows:

$$
\frac{d V}{d t}=-\frac{1}{C_{m}} \times \sum\left[g_{i} \times\left(V-E_{r e v}\right)\right]-I_{a x i a l}
$$

where $V$ is the membrane potential, $C_{\mathrm{m}}$ the membrane capacitance, $g_{i}$ are ionic conductances, and $E_{r e v}$ their respective reversal potentials. The axial flow of current (I axial) between adjacent compartments is calculated by the NEURON simulation package (Hines and Carnevale, 1997). Cytoplasmic resistivity, specific membrane capacitance, and specific membrane resistance were set to $150 \mathrm{Ohm} \cdot \mathrm{cm}, 0.75 \mu \mathrm{F} / \mathrm{cm}^{2}$, and 100,000 $\mathrm{Ohm} \times \mathrm{cm}^{2}$, respectively, with the reversal potential for the leak conductance set at $-50 \mathrm{mV}$. Active conductances followed activationinactivation Hodgkin-Huxley kinetics (Table 1).

Parameters for $\mathrm{I}_{\mathrm{A}}, \mathrm{I}_{\mathrm{Ca}}, \mathrm{I}_{\mathrm{KCa}}$, and $\mathrm{I}_{\mathrm{H}}$ were based on previous published values for SNc DA neurons (Wilson and Callaway, 2000; Amendola et al., 2012; Engel and Seutin, 2015). Fast sodium and potassium currents were derived from Migliore and Schild models, respectively (Schild et al., 1993; Migliore et al., 2008). The SK current is solely activated by an increase in calcium concentration. Therefore, intracellular calcium uptake was modeled as a simple decaying model according to Destexhe et al. (1993). Conductance values were set according to our own measurements or published values (Table 1). Consistent with the literature (Zhou et al., 1998; Kole et al., 2008; Hu et al., 2009; González-Cabrera et al., 2017), $g_{\mathrm{Na}}$ and $\mathrm{g}_{\mathrm{KDR}}$ densities are higher in the AIS than in the rest of the neuron so that AP always initiates in the AIS. According to Gentet and Williams (2007), $\mathrm{I}_{\mathrm{A}}$ density and inactivation kinetics were higher and depolarized, respectively, in the soma compared with the dendritic arbor. Initializing potential was set at $-70 \mathrm{mV}$, and analysis was performed after pacemaking frequency reached a steady state $(8$ spikes). Each simulation run had a $6000 \mathrm{~ms}$ duration with a dt of 0.01 ms. Spatial discretization followed the "d_lambda rule" (Hines and Carnevale, 2001) and adjusted within and between runs when compartment lengths were altered. All dendritic compartments and the axon-start compartment contained all currents, whereas AIS and axon only contained fast sodium and potassium currents $\left(\mathrm{g}_{\mathrm{Na}}, \mathrm{g}_{\mathrm{KDR}}\right)$. Unless otherwise stated, all currents but the fast sodium and potas- 
sium currents had fixed and homogeneous conductance values in the dendrites and the axon-start compartment.

For the realistic morphology models, exact dendrite lengths, soma volume, and diameters of primary dendrites, aDs, axon, and AIS were used (for details, see Immunohistochemistry and morphological reconstruction). The specific branching patterns of each neuron (topology) were also respected. To be consistent with the NEURON software constraints, soma volume was implemented by computing the equivalent cylinder corresponding to the volume measured using "fill out path" method on Simple Neurite Tracer. Axonal diameter was considered constant and set to $0.7 \mu \mathrm{m}$, whereas the diameters of nonprimary dendrites were approximated by a regular tapering to reach a final diameter of $0.5 \mu \mathrm{m}$.

Firing frequency and AP analysis (amplitude, first and second derivative of APs) were computed online by handmade routines directly written in NEURON hoc language (Hines and Carnevale, 2001).

All computing files are available at model DB database under \#245427. Experimental design and statistical analysis. Statistical analysis (performed according to data distribution) included the following: linear regression, unpaired $t$ test, Mann-Whitney, paired $t$ test, and one-way ANOVA, with a $p$ value $<0.05$ being considered statistically significant. Distribution of data was first tested for normality with the Shapiro-Wilk test. Then, the difference between means of two samples was accordingly computed using $t$ tests or Wilcoxon signed rank tests. For comparison between three groups, we used a one-way parametric ANOVA followed by post hoc $t$ tests with Bonferroni correction for multiple comparisons. Linear regressions were obtained with the Pearson test. Data in Figure 12 were fitted using an exponential decay function of equation $f=y_{0}+a \times$ $\exp (-b \times x)$. Unless otherwise stated, statistical data are given as mean \pm $\mathrm{SD}$ and $n$ indicates the number of recorded neurons. Statistical tests were computed by using Sigmaplot 11.0 software (Systat Software) and Prism 6 (GraphPad Software).

Figure preparation. Figures were prepared using Sigma Plot, Prism 6, Adobe Photoshop, and Adobe Illustrator (CS5-CS6, Adobe Systems), Corel Draw X6 (Corel), and ImageJ (J. Schindelin et al., 2012; C. A. Schindelin et al., 2012; Rueden et al., 2017), with brightness and contrast adjustments performed consistently across the images to enhance clarity.

\section{Results}

To investigate the link between morphology and electrophysiological properties in SNc DA neurons, we performed patchclamp recordings combined with post hoc reconstructions of neuronal morphology based on neurobiotin fills of the recorded neurons $(n=40)$. Recordings were obtained from coronal midbrain slices in the presence of synaptic blockers (picrotoxin and kynurenate) to isolate intrinsically generated activity. Recordings and reconstructions were performed on P19-P21 rats (mean age $=$ P20), as both electrophysiological properties and morphological features have been described to reach a mature stage by the end of the second postnatal week (Tepper et al., 1994; Dufour et al., 2014). Although the axon of SNc DA neurons has been previously identified based on its (1) absence of spines, (2) small diameter, and (3) angle of branching from dendrite (Häusser et al., 1995), we performed ankyrinG immunostainings on every recorded neuron to unambiguously identify the axon (Fig. 1).

\section{Variability of axon location and AIS geometry in $\mathrm{SNc}$ DA neurons}

Consistent with the results obtained by Häusser et al. (1995), we observed a high degree of variability in soma-axon distance, with the axon mainly originating from an axon-bearing dendrite (ABD; 35 of 40 cells, $87.5 \%$ ) and not from the soma (Figs. 1, 2). Indeed, the axon often arose from the ABD after the branching of several secondary dendrites (mean number of intermediate secondary dendrites $=1.91, n=35$; Figs. 1,2$)$. We analyzed this unusual configuration by measuring the length and diameters of the $\mathrm{ABD}$ up to the axon $\left(\mathrm{ABD}_{\text {stem }}\right.$ for stem $\left.\mathrm{ABD}\right)$ and the starting diameter of the axon (Fig. $2 A, B$ ). $\mathrm{ABD}_{\text {stem }}$ length ranged from 0 to $106 \mu \mathrm{m}($ mean $=33 \pm 27.5 \mu \mathrm{m}, n=40)$, but the starting and ending diameters of the $\mathrm{ABD}_{\text {stem }}$ did not depend on its length (Fig. 2B). Only the axon-start diameter was weakly negatively correlated with $\mathrm{ABD}_{\text {stem }}$ length $(r=-0.336, p=0.037, n=39)$. We then characterized AIS geometry by measuring the distance between the soma and the AIS (soma-AIS distance), AIS length, and the AIS starting and ending diameters (Fig. 2C). While both soma-AIS distance and AIS length were highly variable, ranging from 20 to $125 \mu \mathrm{m}($ mean $=55 \pm 27 \mu \mathrm{m}, n=40)$ and from 20 to $55 \mu \mathrm{m}$ (mean $=32 \pm 7 \mu \mathrm{m}, n=40$ ), respectively, these two measurements were not correlated (Fig. 2C). Concerning the diameters, the starting diameter of the AIS was negatively correlated with soma-AIS distance $(r=-0.436, p=0.005, n=40)$, while its ending diameter did not show a significant correlation with soma-AIS distance (Fig. 2C). Moreover, as shown in Figure $2 D$, most of the variation in soma-AIS distance was due to variation in $\mathrm{ABD}_{\text {stem }}$ length. Therefore, consistent with previous studies (Häusser et al., 1995; Blythe et al., 2009; GonzálezCabrera et al., 2017; Meza et al., 2018), we found large variations in AIS length, soma-AIS distance, but also in dendrite and axon diameter between SNc DA neurons.

\section{Variability of somatic AP waveform and pacemaking in $\mathrm{SNc}$ DA neurons}

SNc DA neurons in vitro generate a spontaneous regular pattern of activity (low coefficient of variation of the interspike intervals or ISIs) relying on the activity of various somatodendritic and axonal voltage-gated ion channels (Liss and Roeper, 2008; Gantz et al., 2018) (Fig. 3A). The AP in SNc DA neurons is initiated in the axon (Häusser et al., 1995; Blythe et al., 2009), most likely in the AIS, as has been demonstrated in other neuronal types (Kole and Stuart, 2012). Because AIS geometry is highly variable (Fig. 2 ), we wondered whether the shape of the somatic AP was also variable in SNc DA neurons (Fig. 3). SNc DA neurons have been demonstrated to express somatodendritic sodium channels that are responsible for a faithful back-propagation of the AISinitiated AP (Häusser et al., 1995; Seutin and Engel, 2010; Ding et al., 2011a). This property leads to a biphasically rising AP comprising an initial segment (IS) and a somatodendritic (SD) component, representing the contribution of AIS- and SD-located sodium channels, respectively (Grace and Onn, 1989; Häusser et al., 1995; Gentet and Williams, 2007; Blythe et al., 2009; Seutin and Engel, 2010). These two components are particularly easy to separate when computing the first $(\mathrm{dv} / \mathrm{dt})$ and second $\left(\mathrm{d}^{2} \mathrm{v} / \mathrm{dt}^{2}\right)$ time derivatives of the voltage signal during the AP (Fig. $3 A, B$ ). While the first derivative (voltage velocity) is often used by electrophysiologists to analyze AP dynamics (Bean, 2007), we found that the second derivative (voltage acceleration) allows a better discrimination of the IS and SD components (Fig. 3A,B). Although the two derivatives depict different aspects of AP dynamics (voltage velocity and acceleration), we found that the IS and SD peaks of the second derivative are very strongly correlated with their first derivative relatives (Fig. $3 C$ ). To characterize AP shape, we therefore measured AP amplitude and AP half-width but also the amplitudes of the $\mathrm{d}^{2} \mathrm{v} / \mathrm{dt}^{2}$ IS and SD components (Fig. 3D,E). Consistent with previous observations (Amendola et al., 2012; Dufour et al., 2014), both AP amplitude and AP halfwidth displayed significant cell-to-cell variability, ranging from 48 to $78 \mathrm{mV}($ mean $=62 \pm 7.2 \mathrm{mV}, n=54)$ and 0.9 to $1.8 \mathrm{~ms}$ (mean $=1.3 \pm 0.2 \mathrm{~ms}, n=54)$, respectively, but were not correlated (Fig. 3D). Moreover, only the SD component of the $\mathrm{d}^{2} \mathrm{v} / \mathrm{dt}^{2}$ 
A

$\mathrm{ABD}_{\text {stem length }}=0 \mu \mathrm{m}$
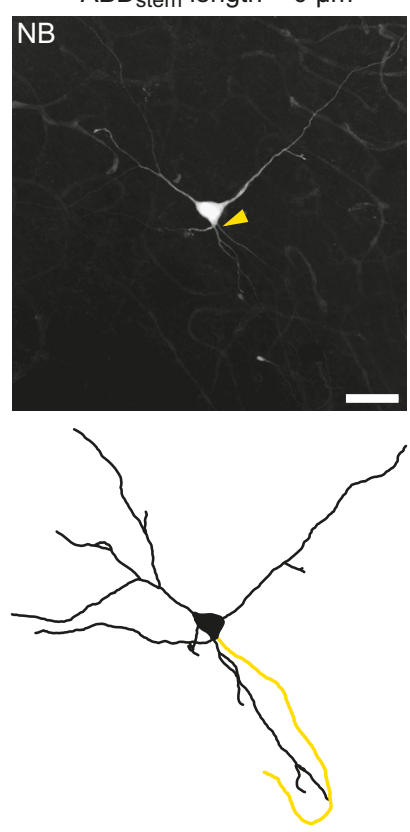

B

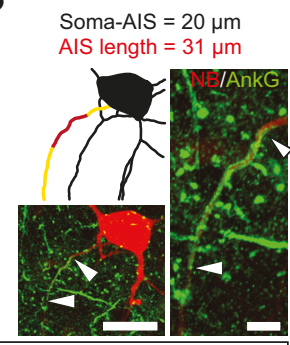

Axon initial segment (AIS)

$\square$ AIS start and end
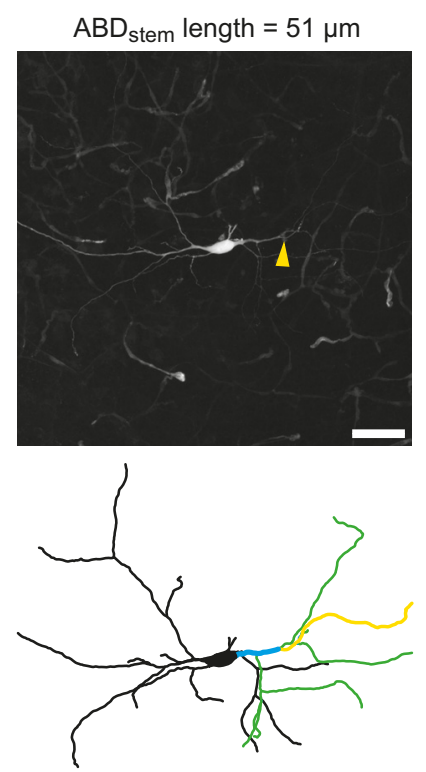

Dxon

stem Axon-bearing dendrite (ABDstem)

- Non axon-bearing dendrite (nABD)

ABD auxiliary dendrite $(\mathrm{aD})$
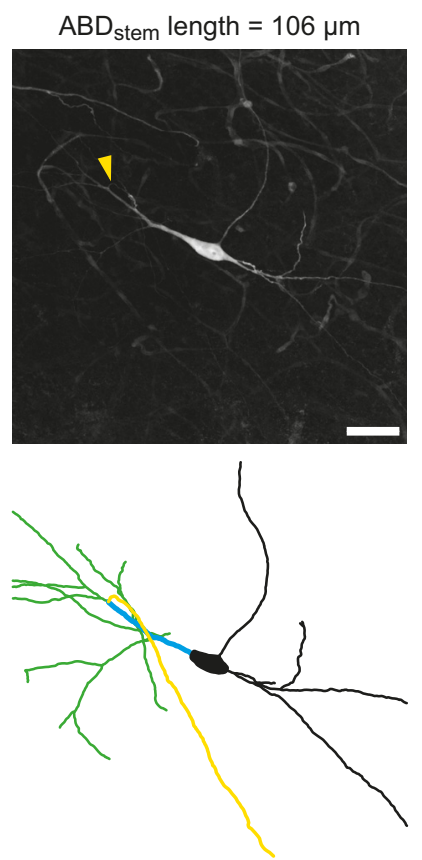

Soma-AIS $=70 \mu \mathrm{m}$ AIS length $=35 \mu \mathrm{m}$
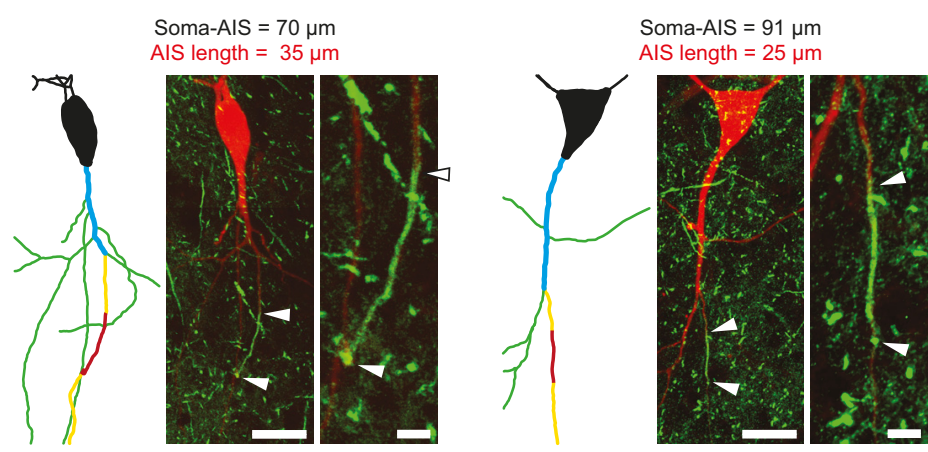

Figure 1. Mature SNc DA neurons display significant cell-to-cell variations in axon-related morphology. $A$, Neurobiotin stainings and skeleton representations of three SNc DA neurons show the variations in the soma-axon distance. While some neurons have a soma-originating axon (left), the axon most often arises from an ABD (middle, right), at distances from the soma up to $100 \mu \mathrm{m}$ (right). Moreover, $A B D$ aDs are often present between the soma and the axon. $\boldsymbol{B}$, Double neurobiotin-ankyrinG stainings and skeleton representations of three SNc DA neurons reveal significant variations in AIS distance from the soma. Each panel represents the skeleton with the original image at the same scale and an expanded version of the immunofluorescence image centered on the AIS. Scale bars: $\boldsymbol{A}, 50 \mu \mathrm{m} ; \boldsymbol{B}, 25 \mu \mathrm{m}$ for the original images, $5 \mu \mathrm{m}$ for the AIS-centered expanded version.

significantly correlated with AP amplitude (Fig. 3E), suggesting that the somatic AP shape is mainly determined by the activity of SD sodium channels.

\section{No relationship between AIS morphology and somatic electrophysiological properties}

Because several studies have demonstrated the influence of AIS geometry on electrophysiological properties recorded at the soma (Kuba et al., 2006; Grubb and Burrone, 2010; Thome et al., 2014; Chand et al., 2015; Hamada et al., 2016; Kole and Brette, 2018), we wondered whether the variability in somatic AP shape could be due to variations in AIS length or distance from the soma. Surprisingly, we found no significant correlation between AIS measurements and AP parameters (Fig. 4): neither AP amplitude nor AP half-width showed correlations with AIS length (AP amplitude vs AIS length, $r=-0.141, p=0.393, n=39$; AP half-width vs AIS length, $r=0.069, p=0.678, n=39$; Fig. $4 B$ ) or distance from the soma (AP amplitude vs soma-AIS distance, $r=$
$0.041, p=0.804, n=39$; AP half-width vs soma-AIS distance, $r=$ $-0.275, p=0.0903, n=39$; Fig. $4 B$ ). While recent studies suggested that the threshold of somatic APs might depend on somaAIS distance (Platkiewicz and Brette, 2010; Thome et al., 2014; Hamada et al., 2016; Kole and Brette, 2018), we did not find a significant correlation between AP threshold and AIS morphology $(r=0.144, p=0.380, n=39)$. The mechanisms underlying the pacemaking in SNc DA neurons are still debated: while several studies highlighted the central role played by somatodendritic conductances (Wilson and Callaway, 2000; Chan et al., 2007; Guzman et al., 2009; Drion et al., 2011; Tucker et al., 2012; Jang et al., 2014), a recent study suggested that the AIS sodium channels may play a major role in controlling pacemaking frequency (Meza et al., 2018). We therefore investigated whether AIS morphology was related to variations in pacemaking frequency. Consistent with previous observations (Matsuda et al., 1987; Putzier et al., 2009; Amendola et al., 2012; Dufour et al., 2014), we observed large cell-to-cell variations in pacemaking 
A

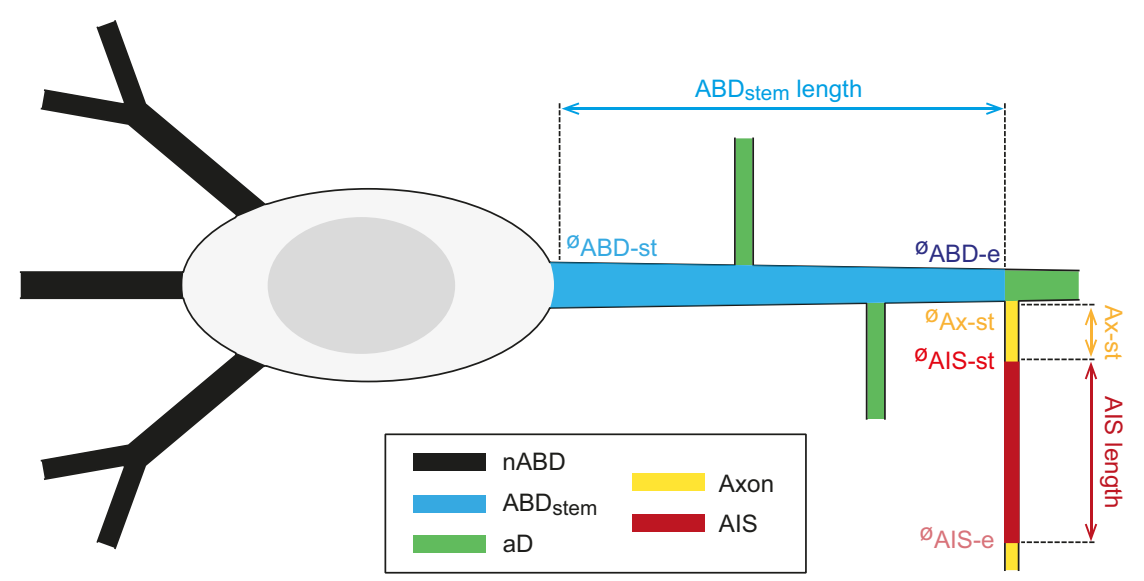

B

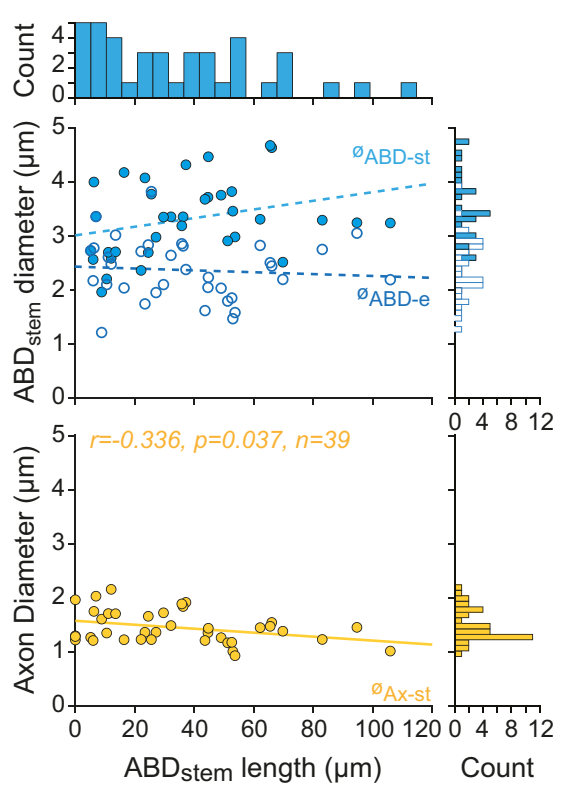

C

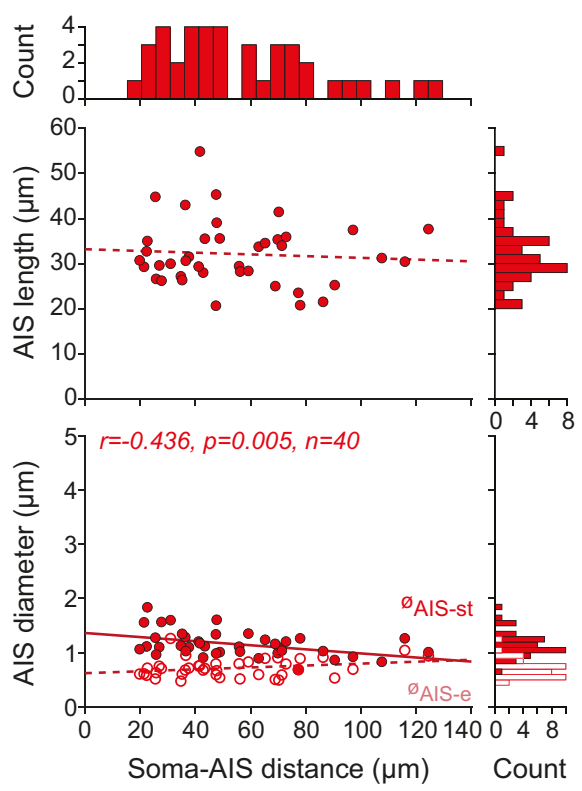

D

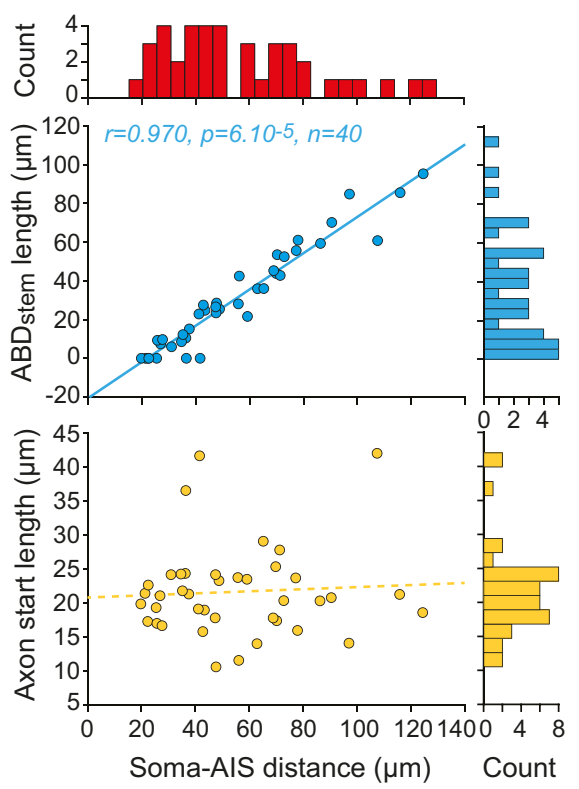

Figure 2. Detailed analysis of cell-to-cell variations in ABD and AIS morphology. $A$, Schematic presenting the main morphological parameters analyzed on each SNC DA neuron. The lengths of the stem $A B D\left(A B D_{\text {stem }}\right)$, the axon start (Ax-st), the AIS (AIS length), the number of aDs, and the number of nABDs were measured. The starting and ending diameters of the stem ABD $\left(\emptyset_{A B D-s t}, \emptyset_{A B D-e}\right)$ and of the AIS $\left(\emptyset_{\text {AIS-st' }}, \emptyset_{\text {AIs-e }}\right)$ as well as the diameter of the axon start $\left(\emptyset_{\text {Ax-st }}\right)$ were also measured. The schematic also corresponds to the average topology observed in the 40 SNC DA neurons from which these measurements were obtained. $B$, Scatter plots representing the variation in $A_{B D}$ stem length and its relationship with $A B D$ (top) or axon diameters (bottom). Histograms represent the distribution of values for each parameter shown above or on the right side of the scatter plots. C, Scatter plots representing the variation in soma-AIS distance and its relationship with AIS length (top) and AIS diameters (bottom). Histograms represent the distribution of values for each parameter shown above or on the right side of the scatter plots. D, Scatter plots representing the variation in soma-AIS distance and its relationship with the length of $\mathrm{ABD}_{\text {stem }}$ (top) or axon start length (bottom). Histograms represent the distribution of values for each parameter shown above or on the right side of the scatter plots. Plain lines indicate significant correlations between parameters. $r, p$, and $n$ values are given on the corresponding plots. Dotted lines indicate nonsignificant correlations.

frequency (from 0.59 to $3.24 \mathrm{~Hz}$, mean $=1.67 \pm 0.73 \mathrm{~Hz}, n=$ 46). However, in the 32 neurons where stable pacemaking was recorded and AIS morphology was analyzed, no significant correlation was found between the spontaneous firing frequency and AIS length or distance from the soma (pacemaking frequency vs AIS length, $r=-0.014, p=0.938, n=32$; pacemaking frequency vs soma-AIS distance, $r=0.005, p=0.980, n=32$; Fig. $4 C$ ).

\section{Role of somatodendritic sodium channels in} AP back-propagation

The results presented so far suggest that AIS morphology does not play a predominant role in shaping SNc DA neuron activity, as neither AP shape nor pacemaking frequency correlates with AIS length or distance from the soma. We wondered whether the reported presence of sodium channels in the dendrites (Häusser et al., 1995), and in particular in the ABD, could explain this lack of dependence. To investigate this point, we first performed double soma/dendrite current-clamp recordings in 29 neurons, which were then labeled and reconstructed to determine whether the recorded dendrites were axon-bearing (ABDs) or non-axonbearing (nABDs; Fig. 5A). These recordings allowed us to monitor the back-propagation of APs from the AIS into the ABD, the soma, and the nABDs. We observed a significant amplification of the AP, particularly in the ABD: while AP amplitude increased between the $\mathrm{ABD}$ and the soma (ABD vs soma; mean $\pm \mathrm{SEM}=$ $58.3 \pm 2.26$ vs $60.6 \pm 2.08 \mathrm{mV}, t=-5.261, p<0.001, n=14$, paired $t$ test), it did not change significantly anymore when the AP propagated into the $\mathrm{nABDs}$ (soma vs $\mathrm{nABD}$; median $=61.4 \mathrm{vs}$ 
A
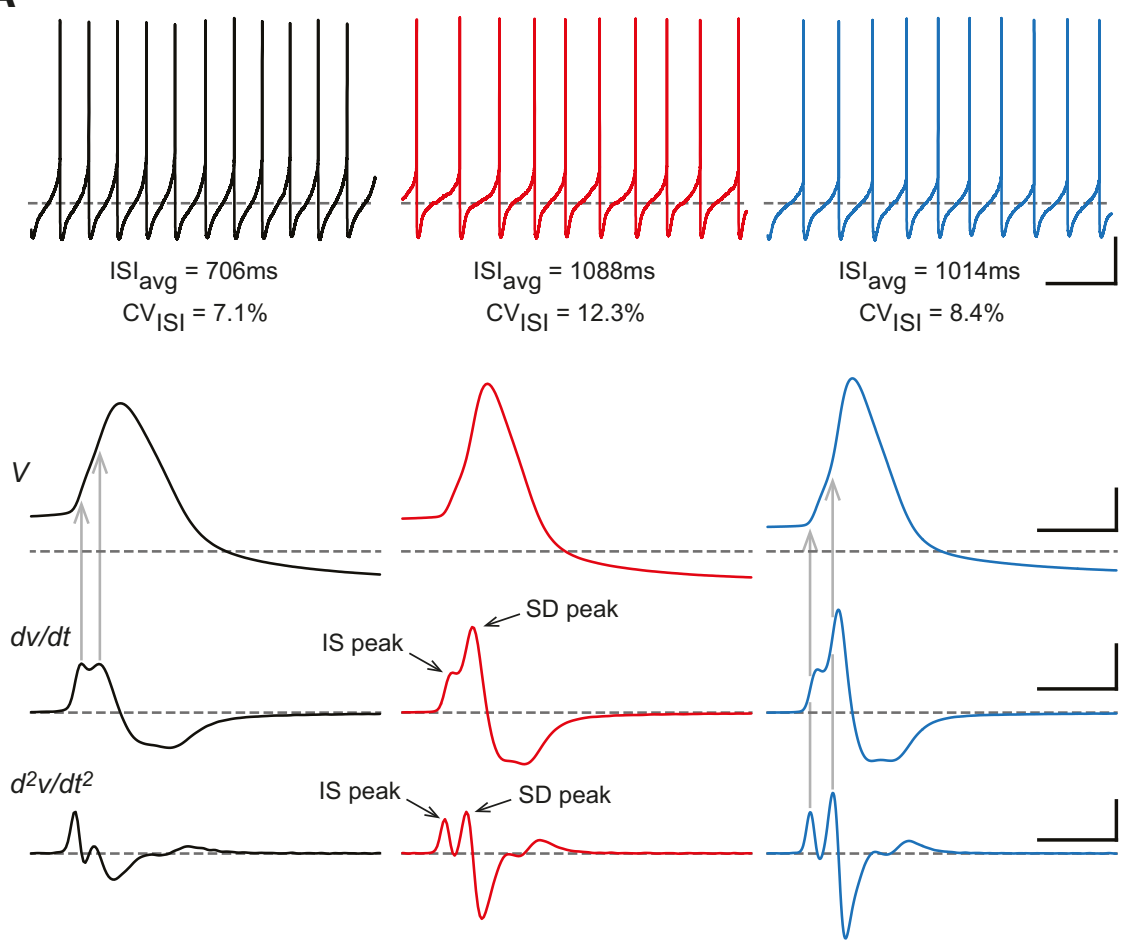

C

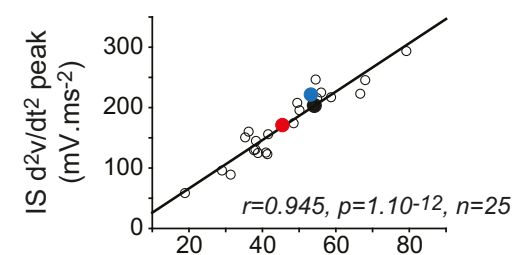

IS dv/dt peak (mV.ms $\left.{ }^{-1}\right)$

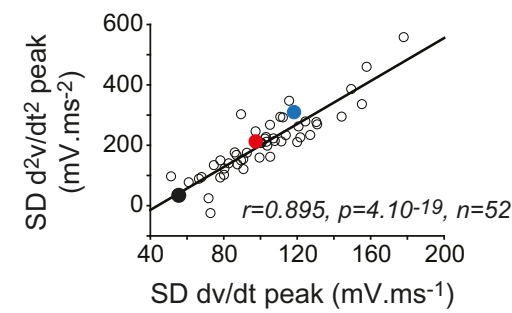

D

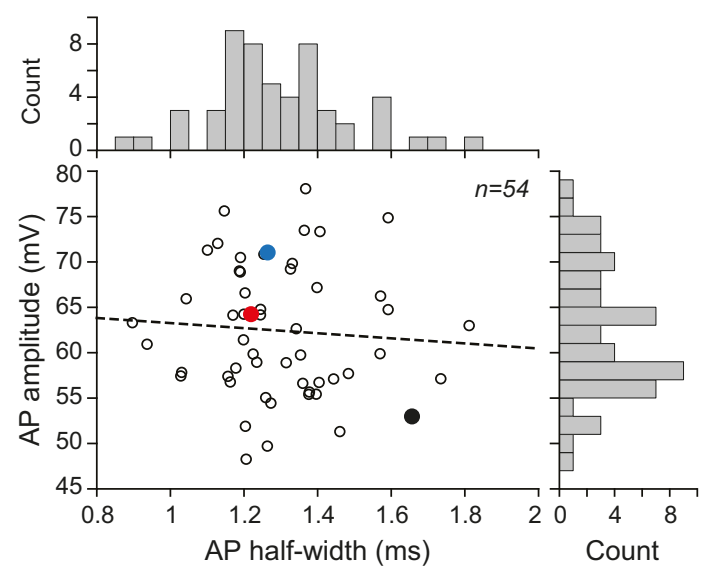

E
B
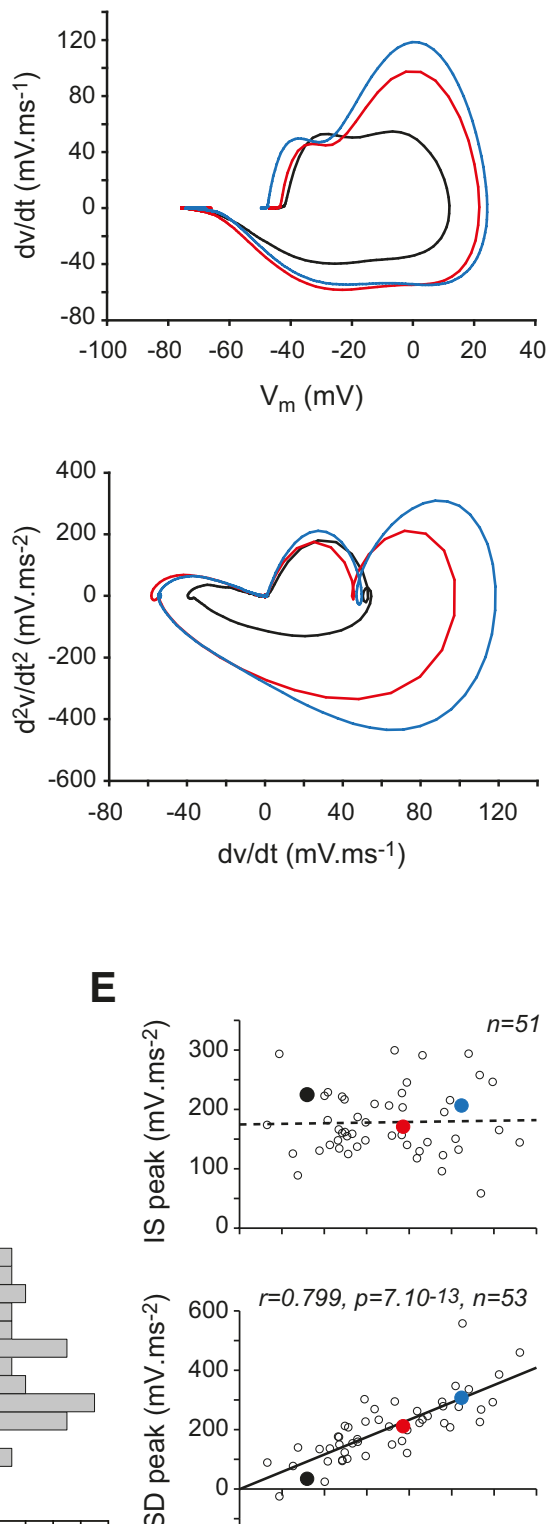

$45 \quad 50 \quad 55 \quad 60 \quad 65 \quad 7075 \quad 80$ AP amplitude $(\mathrm{mV})$

Figure 3. Cell-to-cell variations in soma-recorded AP waveform. $\boldsymbol{A}$, Somatic current-clamp recordings of membrane voltage (top), first time-derivative of voltage (middle), and second time-derivative of voltage (bottom) during an AP in three different neurons. The IS and SD components of the AP are indicated on the center traces for the first and second time-derivatives. Vertical gray arrows indicate the timing of the IS and SD peaks of the first derivative (left traces) or the second derivative (right traces) relative to the AP. $B$, Primary phase-plane plots (dv/dt vs $V_{m}$, top) and secondary phase-plane plot ( $\mathrm{d}^{2} \mathrm{v} / \mathrm{dt}^{2} \mathrm{vs} \mathrm{dv} / \mathrm{dt}$, bottom) for the 3 neurons presented in $A$. C, Top, Scatter plot representing the strong correlation between the IS peak of the second derivative of voltage $\left(\mathrm{d}^{2} \mathrm{v} / \mathrm{dt}^{2}\right)$ and IS peak of the first derivative of voltage (dv/dt) measured in 25 neurons. Bottom, Same type of scatter plot for the SD peak measured in 52 neurons. D, Scatter plot representing the lack of significant correlation between AP amplitude and half-width. The distribution of values for both parameters is shown as histograms on the right and top of the scatter plot. $\boldsymbol{E}$, Scatter plots representing the relationship between the amplitude of the second time-derivative IS or SD component and AP amplitude. Only the SD component was significantly correlated with AP amplitude. Plain lines indicate significant correlations between parameters. $r, p$, and $n$ values are given on the corresponding plots. Dotted lines indicate nonsignificant correlations. Calibration: $A$, Horizontal $2 \mathrm{~s}$ (top), $2 \mathrm{~ms}$ ( 3 bottom rows); vertical $20 \mathrm{mV}$ ( 2 top rows), $50 \mathrm{mV} \cdot \mathrm{ms}^{-1}$ (third row), $200 \mathrm{mV} \cdot \mathrm{ms}^{-2}$ (bottom row). Horizontal gray dotted lines indicate $-60 \mathrm{mV}$ (voltage traces) and $0 \mathrm{mV} \cdot \mathrm{ms}{ }^{-1}$ or $0 \mathrm{mV} \cdot \mathrm{ms}^{-2}$ for the derivative traces. $C-E$, Values corresponding to the recordings presented in $A$ are identified by colored dots of matching colors.

61.7, $z=0.454, p=0679, n=15$, Wilcoxon signed rank test; Fig. $5 B)$. Consistently, AP half-width decreased between the ABD and the soma ( $\mathrm{ABD}$ vs soma; mean $\pm \mathrm{SEM}=1.24 \pm 0.06$ vs $1.14 \pm$ $0.06 \mathrm{~ms}, t=5.632, p<0.001, n=14$, paired $t$ test) but did not change significantly between the soma and the nABDs (soma vs $\mathrm{nABD}$; mean $\pm \mathrm{SEM}=1.31 \pm 0.06$ vs $1.30 \pm 0.06 \mathrm{~ms}, t=1.441$, $p=0.172, n=15$, paired $t$ test; Fig. $5 B$ ). The analysis of the IS and SD components of the $\mathrm{d}^{2} \mathrm{v} / \mathrm{dt}^{2}$ confirmed and extended these observations: while the IS component displayed a strong decrease between the $\mathrm{ABD}$ and the soma (ABD vs soma; mean $\pm \mathrm{SEM}=$ $595 \pm 46$ vs $274 \pm 31 \mathrm{mV} \cdot \mathrm{ms}^{-2}, t=7.47, p<0.001, n=14$, paired $t$ test), the SD component displayed a strong increase in amplitude over the same course (ABD vs soma; mean \pm SEM $=$ $185 \pm 49$ vs $342 \pm 60 \mathrm{mV} \cdot \mathrm{ms}^{-2}, t=-4.284, p<0.001, n=13$, paired $t$ test; Fig. $5 C$ ). Although of smaller magnitudes, these changes persisted when the AP propagated into the nABDs (IS 
A
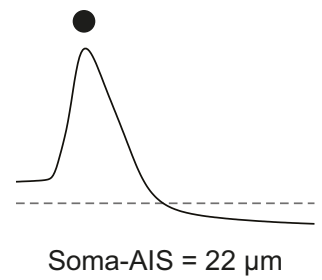

AIS length $=33 \mu \mathrm{m}$
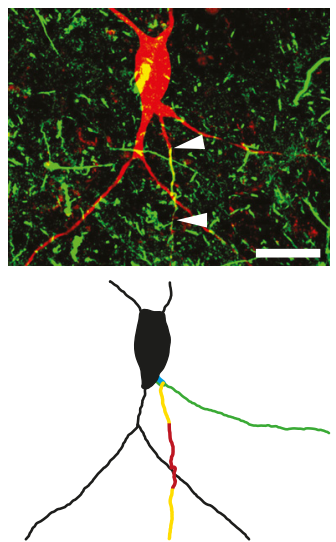

B
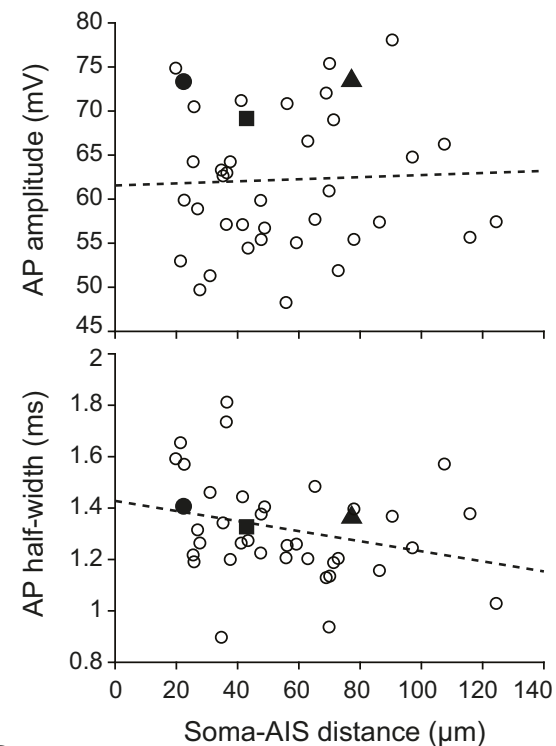

C

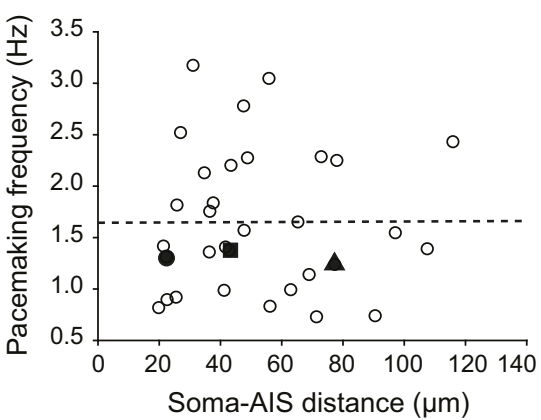

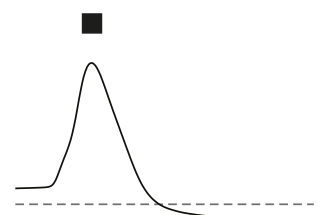

Soma-AIS $=43 \mu \mathrm{m}$ AIS length $=28 \mu \mathrm{m}$
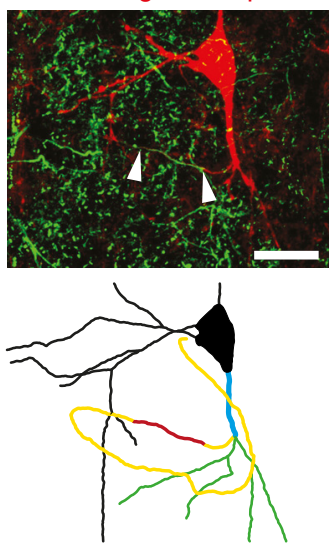

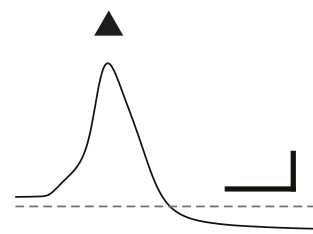

Soma-AIS $=77 \mu \mathrm{m}$ AIS length $=23 \mu \mathrm{m}$
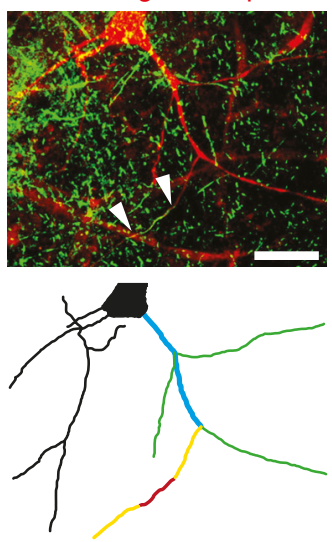
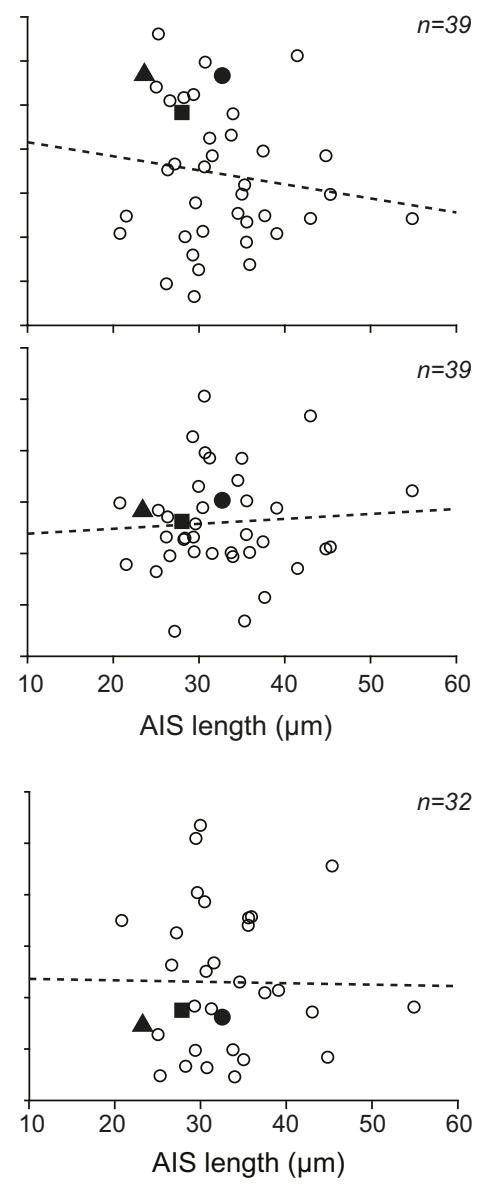

Figure 4. Absence of correlation between electrophysiological parameters and AIS geometry. $\boldsymbol{A}$, Top, Somatic current-clamp recordings of the AP in 3 neurons presenting significant variations in soma-AIS distance and AIS length. Middle, Double immunolabeling (neurobiotin/ankyrinG; red/green) of the recorded neurons showing AIS location and length. White arrowheads indicate AIS start and end. Bottom, Skeleton representation of the same neurons indicates the axon, AIS, ABD, and aDs, using the color coding already used in Figures 1 and 2. B, Scatter plots representing the lack of significant correlations between soma-AIS distance (left) or AIS length (right) and AP amplitude (top) or half-width (bottom) observed in 39 recorded and reconstructed neurons. $\boldsymbol{C}$, Scatter plots representing the lack of significant correlations between soma-AIS distance (left) or AIS length (right) and pacemaking

soma vs IS nABD, mean $\pm \mathrm{SEM}=374 \pm$ 27 vs $264 \pm 20 \mathrm{mV} \cdot \mathrm{ms}^{-2}, t=5.86, p<$ $0.001, n=13$, paired $t$ test; SD soma vs SD $\mathrm{nABD}$, median $=212$ vs $254 \mathrm{mV} \cdot \mathrm{ms}^{-2}$, $z=3.181, p<0.001, n=15$, Wilcoxon signed rank test; Fig. 5C).

Density of somatodendritic sodium and delayed rectifier potassium currents

To determine whether this profile of AP back-propagation arose from a differential distribution of sodium channels in the $\mathrm{ABD}$, soma, and nABDs, we measured sodium currents in these different compartments using outside-out patch-clamp recordings (Fig. 6A-C). While we observed a large variability in sodium conductance density $\left(\mathrm{g}_{\mathrm{Na}}\right)$, ranging from 29 to $180 \mathrm{pS} / \mu \mathrm{m}^{2}$ $\left(\right.$ mean $\left.=70 \pm 45 \mathrm{pS} / \mu \mathrm{m}^{2}, n=29\right)$, conductance density did not seem to depend on the recording site ( $\mathrm{ABD}$, soma or $\mathrm{nABDs}$ ), as revealed by the lack of significant correlation between $\mathrm{g}_{\mathrm{Na}}$ and pipette-soma distance or pipette-AIS distance (Fig. 6B). Although other voltage-gated currents have been demonstrated to display heterogeneous distributions in the SD compartment of SNc DA neurons (Gentet and Williams, 2007; Dufour et al., 2014; Engel and Seutin, 2015), our results suggest a fairly homogeneous pattern of expression of sodium channels in the SD compartment. We then wondered whether the variability in sodium conductance density could be related to cell-to-cell variations in soma-AIS distance (Fig. 6C): no correlation was found between somaAIS distance and sodium conductance density.

AP shape is not only defined by sodium currents but also by potassium currents, in particular the delayed rectifier type, which play a major role in the control of AP halfwidth (Lien and Jonas, 2003; Bean, 2007; Ding et al., 2011b). Using the same type of recordings as for sodium currents, we measured delayed rectifier potassium currents in the ABD, soma, and nABDs of SNc DA neurons (Fig. 6D-F). Again, while a significant variability in potassium conductance $\left(\mathrm{g}_{\mathrm{KDR}}\right)$ was observed, ranging from 11 to $626 \mathrm{pS} / \mu \mathrm{m}^{2}\left(\right.$ mean $=151 \pm 165 \mathrm{pS} / \mu \mathrm{m}^{2}$, $n=25$ ), no significant relationship was found with any of the morphological parameters analyzed: potassium conductance density was independent of recording loca-

\section{$\leftarrow$}

frequency observed in 32 recorded and reconstructed neurons. $B, C$, Values corresponding to the 3 neurons presented in $A$ are identified by a black circle, square, or triangle. Calibration: $\boldsymbol{A}$, recordings, $20 \mathrm{mV}$ vertical, $2 \mathrm{~ms}$ horizontal; pictures, $25 \mu \mathrm{m}$. Horizontal dotted lines indicate $-60 \mathrm{mV}$. B, C, Dotted lines indicate linear regressions (nonsignificant in all cases). 
A

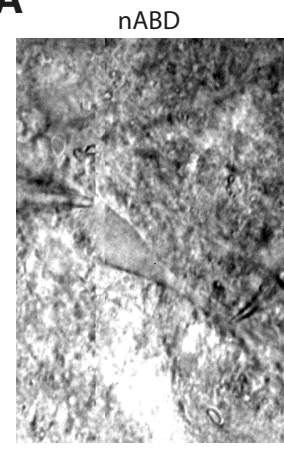

Pipette to soma $=21 \mu \mathrm{m}$
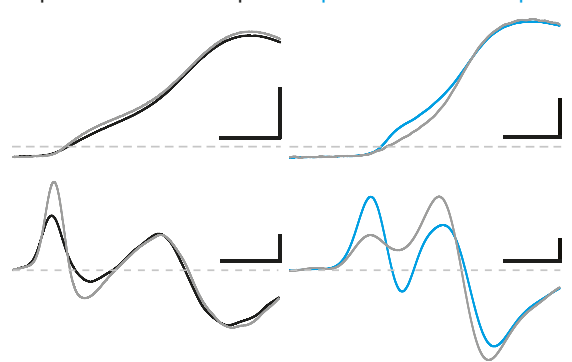

C
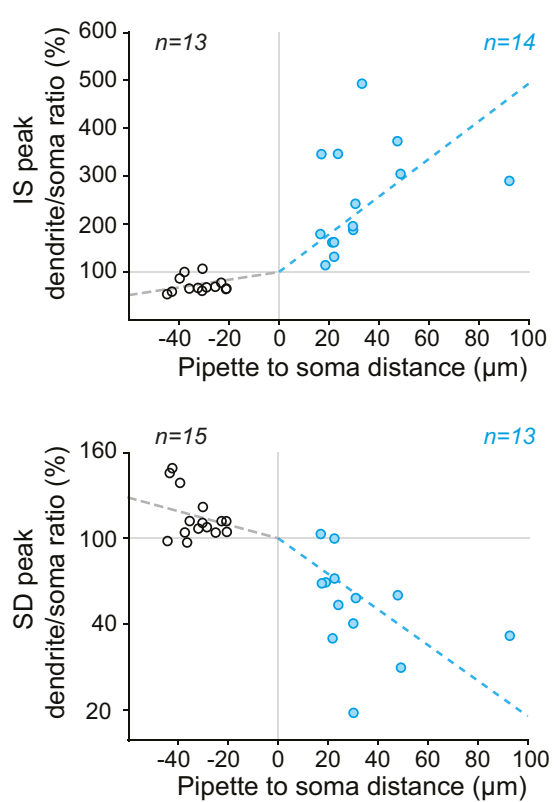

B
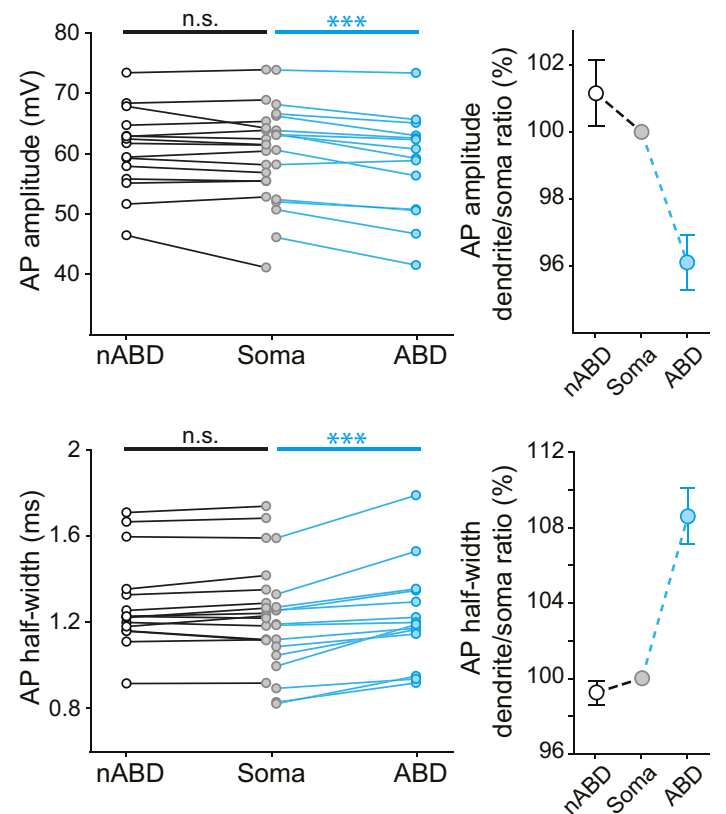
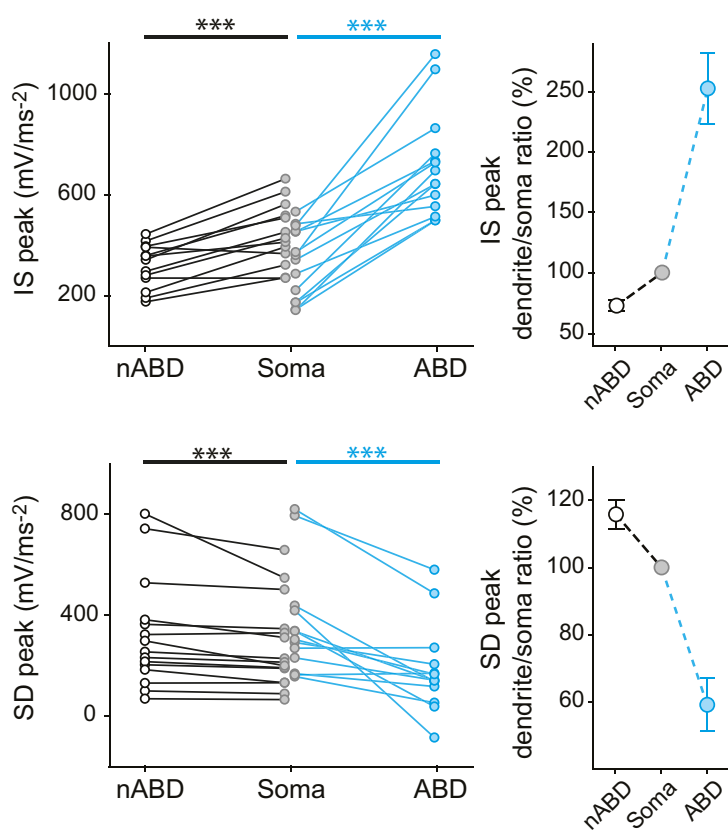

Figure 5. Back-propagating spikes suggest the presence of significant Na channel density in dendrites. $A$, Top, Photomicrographs showing examples of double recordings from a $\mathrm{ABBD}$ (left) and an ABD (right). Middle, Somatic (gray trace) and dendritic (black or blue traces) current-clamp recordings of the same neurons. Bottom, Second time derivative of the voltage $\left(\mathrm{d}^{2} \mathrm{v} / \mathrm{dt}^{2}\right)$ during the AP in the same neurons. $B$, Scatter plots representing the changes in AP amplitude (top) and half-width (bottom) during the back-propagation of an AP in the ABD, soma, and nABD. Right, Values normalized to soma. C, Scatter plots representing the changes in IS and SD d ${ }^{2} \mathrm{v} / \mathrm{dt}^{2}$ components during back-propagation. Left, IS (top) and SD (bottom) amplitudes normalized to soma represented as a function of the recording distance from the soma. Positive distances correspond to ABD recordings, and negative distances correspond to nABD recordings. Middle, Changes in IS (top) and SD (bottom) raw amplitudes between ABD, soma, and nABDs. Right, Changes in normalized IS and SD components in ABD, soma, and nABDs. Dotted lines indicate nonsignificant correlations. Normalized data on the right scatter plots are indicated as mean \pm SEM. ${ }^{* * *} p<0.001$, n.s. not statistically significant. Calibration: $A$, Pictures, $20 \mu \mathrm{m}$. Recordings, $20 \mathrm{mV}$ vertical, $0.25 \mathrm{~ms}$ horizontal (middle left); $20 \mathrm{mV}$ vertical, $0.5 \mathrm{~ms}$ horizontal (middle right), $200 \mathrm{mV} \cdot \mathrm{ms}^{-2}$ vertical, $0.25 \mathrm{~ms}$ horizontal (bottom left), $50 \mathrm{mV} \cdot \mathrm{ms}^{-2}$ vertical, $0.5 \mathrm{~ms}$ horizontal (bottom right). Horizontal gray dotted lines indicate $-40 \mathrm{mV}$ (voltage traces) and $0 \mathrm{mV} \cdot \mathrm{ms}^{-2}$ (derivative traces).

tion, distance from the AIS, or soma-AIS distance (Fig. 6E,F). Overall, these results show that sodium and delayed rectifier potassium currents are expressed in the SD compartment of SNc DA neurons in a fairly homogeneous manner, while displaying significant cell-tocell variability in conductance density.

In summary, our experimental results suggest that, in SNc DA neurons, AIS morphology shows significant cell-to-cell varia- tions. In particular, the axon most often arises from an $\mathrm{ABD}$ of highly variable length and complexity. Despite this, AIS morphology seems to have a negligible impact on the shape of the AP recorded at the soma or on pacemaking frequency. Moreover, dendritic recordings indicate that sodium and delayed rectifier potassium currents are fairly homogeneously expressed in the soma and primary dendrites of SNc DA neurons. To determine 


\section{A}

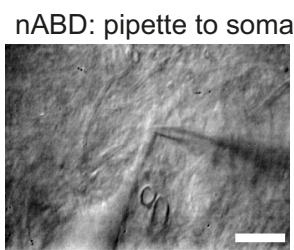

$-14 \mu \mathrm{m}$
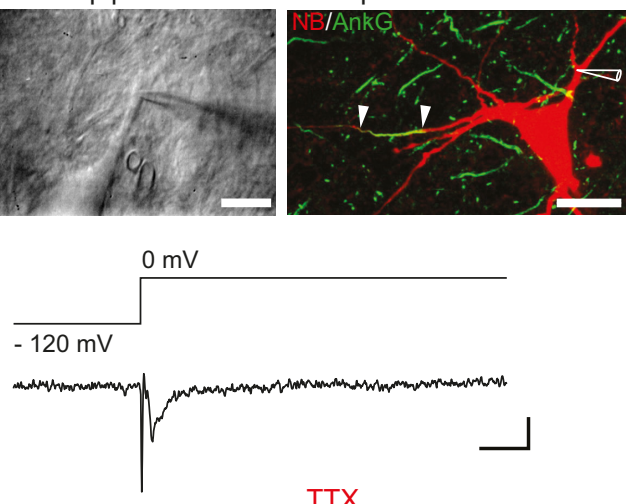

TTX

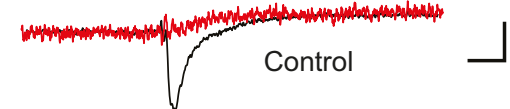

B
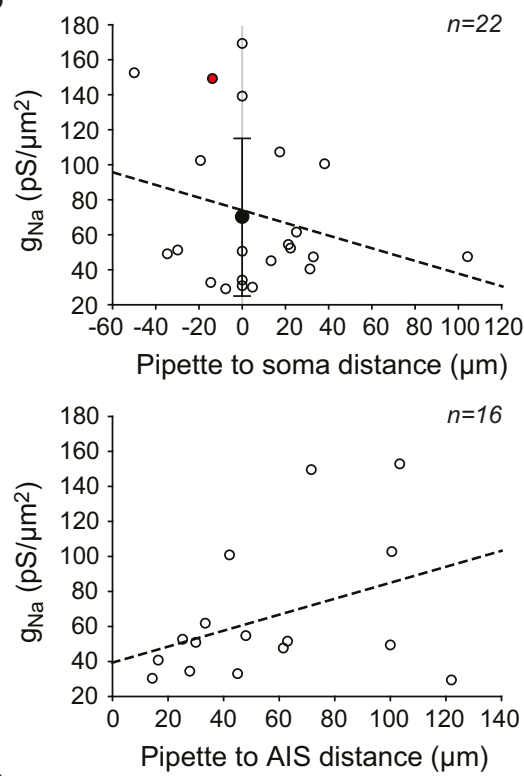

C

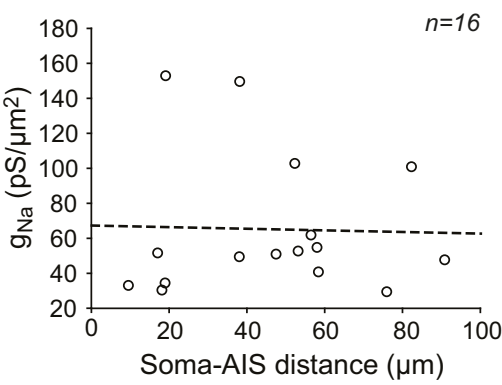

D
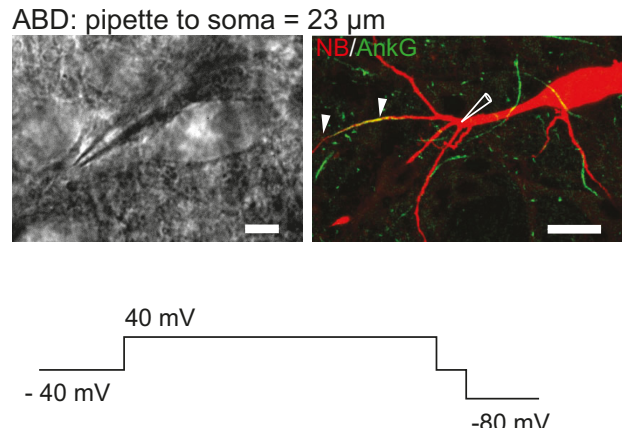

E
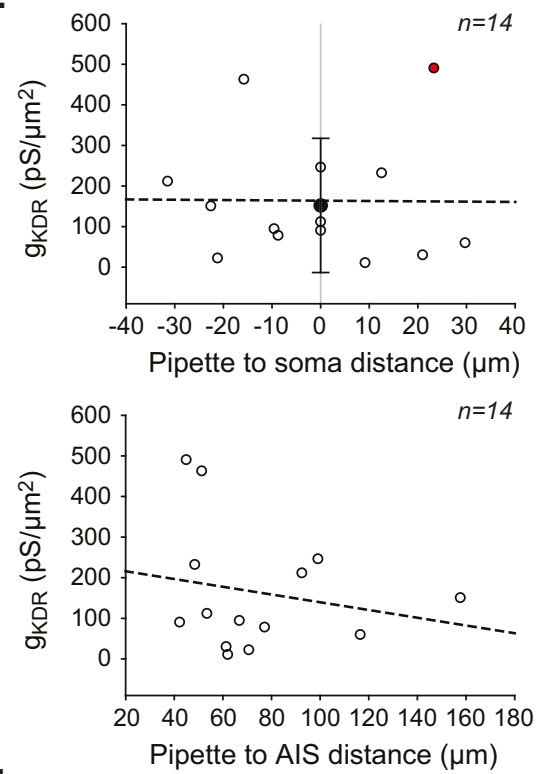

F
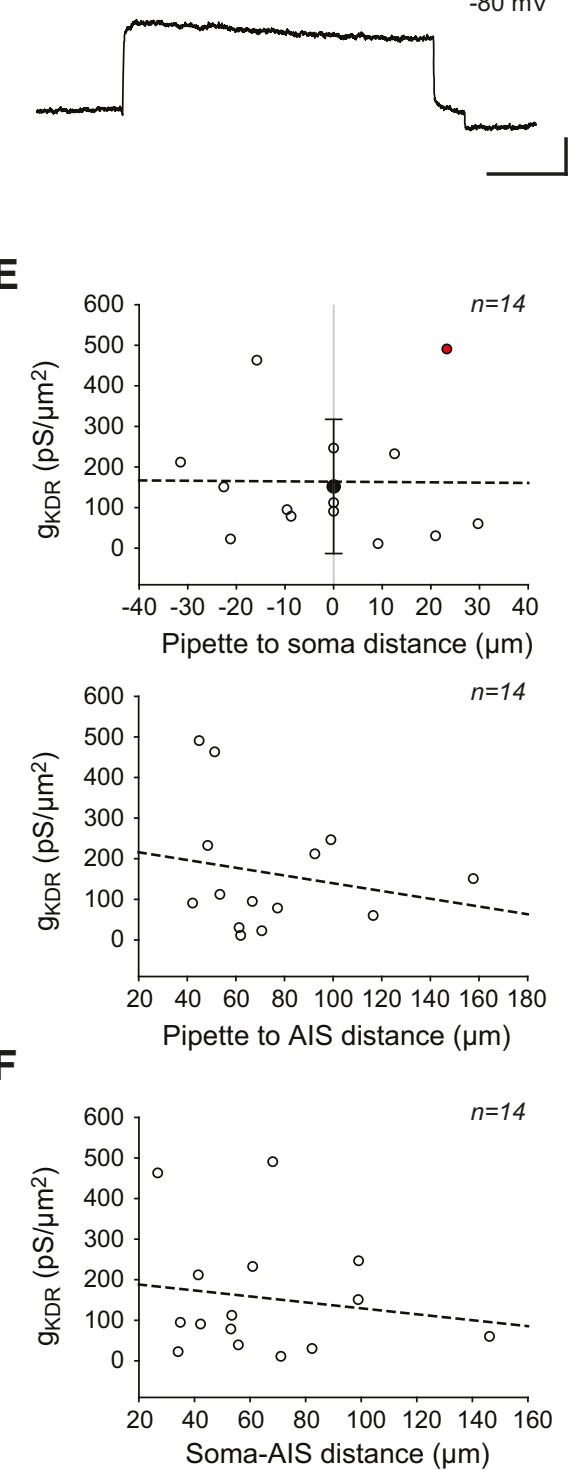

Figure 6. Somatodendritic sodium and delayed rectifier potassium current recordings. $A$, Top left, Photomicrograph showing an example of a recording from a nABD. Top right, Same neuron labeled for neurobiotin (red) and ankyring (green) with the recording location indicated by a white schematized pipette. White arrows indicate the AIS start and end. Middle, Outside-out recording of the sodium current corresponding to the neuron shown above. Bottom, Somatic outside-out recording from another neuron using the same protocol showing the sensitivity of the sodium current to TTX. B, Scatter plots representing the lack of significant correlations between sodium conductance density and the distance of recording site from the soma (top) or the AIS (bottom). Top, Negative and positive distance values correspond to $\mathrm{nABD}$ and $\mathrm{ABD}$ recording sites, respectively. The value corresponding to the neuron presented in $A$ is indicated by a red dot. Black circle represents the average conductance density. Error bars indicate SD. C, Scatter plot representing the lack of correlation between sodium conductance density and soma-AIS distance. Dotted lines indicate nonsignificant correlations. $\boldsymbol{D}-\boldsymbol{F}$, Description of the delayed rectifier current in the somatodendritic compartment using the same presentation as the one given for sodium currents in $\boldsymbol{A}-\boldsymbol{C} . \boldsymbol{D}$, Neuron where an outside-out recording was obtained from the ABD. Calibration: $A$, $\boldsymbol{D}$, Top left, $10 \mu \mathrm{m}$; Top right, $25 \mu \mathrm{m}$. $A$, Middle, Bottom, $10 \mathrm{pA}$ vertical, $2 \mathrm{~ms}$ horizontal; $\boldsymbol{D}$, Bottom, $50 \mathrm{pA}$ vertical, 25 ms horizontal. 
how these peculiar biophysical and morphological characteristics interact to influence AP shape and firing frequency, we built a multicompartment model of SNc DA neuron based on the morphological reconstructions presented above.

\section{Influence of variable axonal and dendritic sodium channel density on activity}

Our multicompartment model included most of the somatodendritic voltage- and calcium-gated ion currents known to be involved in pacemaking (Liss and Roeper, 2008; Drion et al., 2011; Gantz et al., 2018): $\mathrm{I}_{\mathrm{H}}, \mathrm{I}_{\mathrm{CaL}}(\mathrm{Cav1.3}), \mathrm{I}_{\mathrm{A}}, \mathrm{I}_{\mathrm{SK}}, \mathrm{I}_{\mathrm{Na}}$, and $\mathrm{I}_{\mathrm{KDR}}$ (Table 1). The axon contained only $\mathrm{I}_{\mathrm{Na}}$ and $\mathrm{I}_{\mathrm{KDR}}$, with a higher density of these currents in the AIS than in the rest of the axon, consistent with observations made in several neuronal types (Clark et al., 2009; Kole and Stuart, 2012). As a first step, we used an averagemorphology model (Fig. 7A) to determine which densities of dendritic and axonal $\mathrm{I}_{\mathrm{Na}}$ and $\mathrm{I}_{\mathrm{KDR}}$ could replicate the electrophysiological profile recorded in real neurons (AP shape, amplitudes of IS and SD d ${ }^{2} \mathrm{v} / \mathrm{dt}^{2}$ components, faithful back-propagation). The variations in SD conductance densities were based on the outside-out voltage-clamp recordings presented in Figure 6: we thus chose to vary $\mathrm{g}_{\mathrm{Na}}$ from 25 to $200 \mathrm{pS} / \mu \mathrm{m}^{2}$ ( $25 \mathrm{pS}$ increment) and $\mathrm{g}_{\mathrm{KDR}}$ from 50 to $400 \mathrm{pS} / \mu^{2} \mathrm{~m}^{2}$ (50 $\mathrm{pS}$ increment). For simplicity, we systematically covaried these two conductances, keeping their ratio constant $\left(\mathrm{g}_{\mathrm{Na}} / \mathrm{g}_{\mathrm{KDR}}=0.5\right)$. Except for the lowest pair of values of densities $\left(25 / 50 \mathrm{pS} / \mu \mathrm{m}^{2}\right.$ for $\left.\mathrm{g}_{\mathrm{Na}} / \mathrm{g}_{\mathrm{KDR}}\right)$, which generated unstable electrophysiological behaviors in some neurons (data not shown), this range of SD densities yielded amplitudes of the $\mathrm{SD} \mathrm{d} \mathrm{d}^{2} \mathrm{v} / \mathrm{dt}^{2}$ component at the soma very similar to the ones observed in our recordings ( $30-450$ vs $0-500 \mathrm{mV} \cdot \mathrm{ms}^{-2}$; Fig. $7 B$ vs Fig. $3 E$ ). For the AIS, we determined $\mathrm{g}_{\mathrm{Na}}$ such that the amplitudes of the IS $\mathrm{d}^{2} \mathrm{v} / \mathrm{dt}^{2}$ component matched the range of experimental values observed in our recordings $\left(100-300 \mathrm{mV} \cdot \mathrm{ms}^{-2}\right)$. The AIS $\mathrm{g}_{\mathrm{Na}}$ densities defined by this constraint ranged from 1000 to 8000 $\mathrm{pS} / \mu \mathrm{m}^{2}$ (1000 pS increment; Fig. $\left.7 A, B\right)$. As $\mathrm{g}_{\mathrm{KDR}}$ in the AIS is unknown, we chose to couple it to $\mathrm{g}_{\mathrm{Na}}$ using the same range of values $\left(1000-8000 \mathrm{pS} / \mu \mathrm{m}^{2}\right)$. The differences in densities between the AIS and the SD compartment are consistent with the reported high density of sodium channels in the AIS compared with soma and dendrites (Kole et al., 2008; Lorincz and Nusser, 2010). All other conductance densities were kept constant (Table 1).

Our model reproduced the typical electrophysiological features observed in real SNc DA neurons (Fig. 7C): a spontaneous pacemaking pattern of activity, a voltage sag and a biphasic rebound in response to hyperpolarizing current pulses, and a broad biphasic AP characteristic of SNc DA neurons (Grace and Bunney, 1984; Washio et al., 1999; Seutin and Engel, 2010; Amendola et al., 2012; Dufour et al., 2014). We then wondered whether the model was able to reproduce the specific profile of AP backpropagation described in Figure 5. Most combinations of SD and AIS $\mathrm{g}_{\mathrm{Na}}$ densities reproduced the increases in AP amplitude and in $\mathrm{SD} \mathrm{\textrm {d } ^ { 2 }} \mathrm{v} / \mathrm{dt}^{2}$ component and the decreases in AP duration and in IS $\mathrm{d}^{2} \mathrm{v} / \mathrm{dt}^{2}$ component observed between the ABD, soma, and $\mathrm{nABDs}(p<0.001$ for all significant differences, ANOVA test with Bonferroni correction; Fig. $8 B, C$ ). The combinations of $50-$ $100 \mathrm{pS} / \mu \mathrm{m}^{2}$ and $3000-5000 \mathrm{pS} / \mu \mathrm{m}^{2}$ for SD and AIS $\mathrm{g}_{\mathrm{Na}}$, respectively, quantitatively best reproduced the back-propagation profile (Fig. $8 \mathrm{C}$ vs Fig. 5). The model also reproduced the strong link found between AP amplitude and SD d $\mathrm{d}^{2} \mathrm{v} / \mathrm{dt}^{2}$ and the absence of link between AP amplitude and IS $\mathrm{d}^{2} \mathrm{v} / \mathrm{dt}^{2}$, independent of the values of SD and AIS $g_{\mathrm{Na}}$ (Fig. $8 D$ ). Moreover, the range of AP amplitudes obtained matched the experimentally observed values ( $52-88 \mathrm{mV}$ vs $48-78 \mathrm{mV}$; Fig. $8 D$ vs Fig. $3 E$ ). Our model is therefore able to faithfully reproduce the two phases of the AP (IS and SD), their specific back-propagation profiles, and the changes in amplitudes and duration observed between the ABD, the soma, and the nABDs (Fig. 8B,E).

We then tried to determine the respective influence of $S D$ and AIS $g_{\text {Na }}$ on pacemaking frequency in our constrained model (Fig. $8 F)$. Interestingly, while varying $\mathrm{SD} \mathrm{g}_{\mathrm{Na}}$ over its full range increased pacemaking frequency by as much as $35 \%$ (from 1.79 to $2.41 \mathrm{~Hz}$ ), varying $\mathrm{g}_{\mathrm{Na}}$ in the AIS increased pacemaking frequency by $<6 \%$ (from 1.79 to $1.88 \mathrm{~Hz}$ ). Therefore, our model suggests that pacemaking frequency in SNc DA neurons is more strongly controlled by SD than by AIS sodium channels.

\section{Influence of variable dendrite and AIS morphology on activity}

While these simulations were performed using an average morphology, our experiments demonstrated important cell-to-cell variations in morphology, at both the dendritic and axonal levels (Fig. 2). As AIS length has been reported to influence excitability in several neuronal types (Kuba et al., 2006, 2014; Gutzmann et al., 2014), including mouse SNc DA neurons (Meza et al., 2018), we first tested how variations in AIS length modified pacemaking frequency in a simplified version of our model (no intermediate dendrites on the ABD; Fig. 9A,B). All combinations of SD and AIS $g_{\mathrm{Na}}$ were tested, but we present the results only for $50-100$ $\mathrm{pS} / \mu \mathrm{m}^{2}$ in the dendrites and $3000-5000 \mathrm{pS} / \mu \mathrm{m}^{2}$ in the AIS (the other combinations gave very similar results). While modifying AIS $\mathrm{g}_{\mathrm{Na}}$ density had little influence on pacemaking frequency $(<6 \%$; Fig. $8 F)$, we found that pacemaking frequency was more strongly influenced by modifying AIS length (Fig. $9 \mathrm{~B}$ ): changing the length from 20 to $60 \mu \mathrm{m}$ increased frequency by as much as $17.5 \%$ (from 1.13 to $1.33 \mathrm{~Hz}$ ), depending on SD and AIS $\mathrm{g}_{\mathrm{Na}}$ densities. Using the same simplified model, we then tested whether a variable soma-AIS distance was associated with significant variations in pacemaking frequency (Fig. $9 C, D$ ), as AIS distance has also been proposed to influence neuronal excitability (Grubb and Burrone, 2010; Hamada et al., 2016). Because most of the cell-to-cell variation in soma-AIS distance is indeed due to variations in $A B D$ length (Fig. $2 D$ ), we varied $A B D_{\text {stem }}$ length from 5 to $80 \mu \mathrm{m}$ while keeping the axon-start length constant at $20 \mu \mathrm{m}$. This morphological variation induced increases in pacemaking frequency similar to the ones observed when varying AIS length (maximum 15\%, from 1.07 to $1.23 \mathrm{~Hz}$; Fig. 9D).

However, it has to be noted that cell-to-cell variations in $\mathrm{ABD}_{\text {stem }}$ length are associated with a significant increase in the number of secondary dendrites branching from the $\mathrm{ABD}$ in between the soma and the axon (aDs; Fig. 10A). In addition, dendritic topology has been shown to greatly influence neuronal output (Mainen and Sejnowski, 1996; Vetter et al., 2001; van Ooyen et al., 2002). To take these findings into account, we next tested the influence of varying $\mathrm{ABD}_{\text {stem }}$ length with the associated change in dendritic complexity (Fig. 10B-D). Interestingly, including aDs greatly increased the influence of soma-AIS distance on pacemaking frequency: changing this distance from $25 \mu \mathrm{m}$ (with 1 $\mathrm{aD})$ to $100 \mu \mathrm{m}$ (with $5 \mathrm{aDs}$ ) increased pacemaking frequency by $>100 \%$ (from 1.07 to $2.24 \mathrm{~Hz}$; Fig. 10C). Moreover, while the increase in frequency due to dendritic complexity was strongly potentiated by changes in $\mathrm{SD} \mathrm{g}_{\mathrm{Na}}$ density, it was almost insensitive to changes in AIS $\mathrm{g}_{\mathrm{Na}}$ (Fig. 10D). We then wondered whether the increase in ABD complexity modified the impact of AIS length on pacemaking frequency. Indeed, while varying AIS length in the simplest model $(1 \mathrm{aD})$ increased pacemaking fre- 


\section{A}

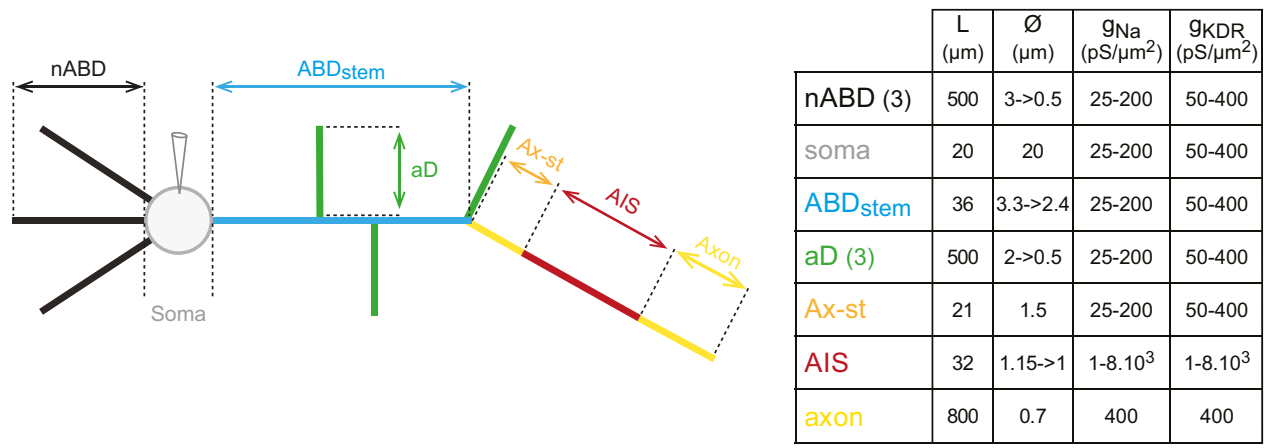

B
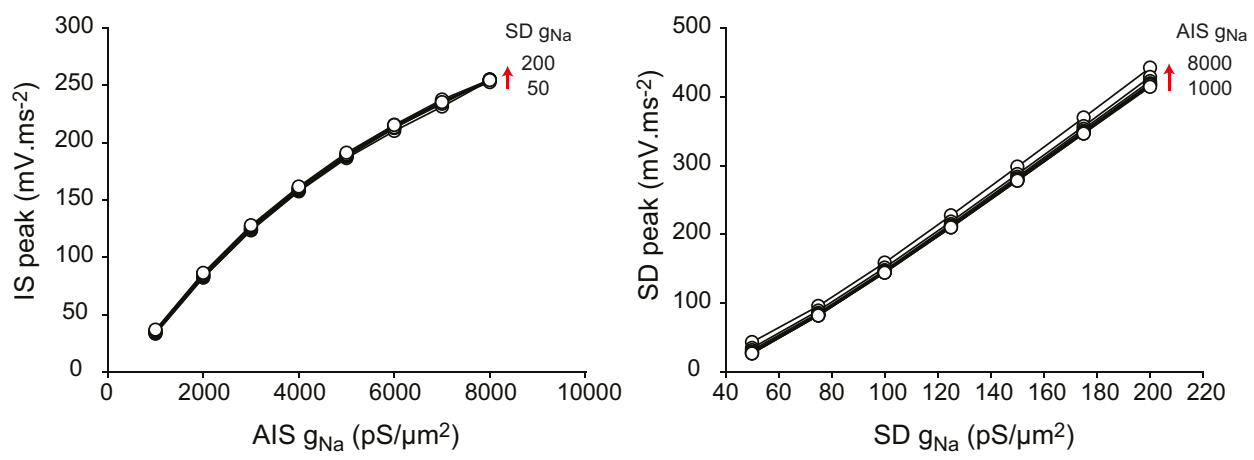

C
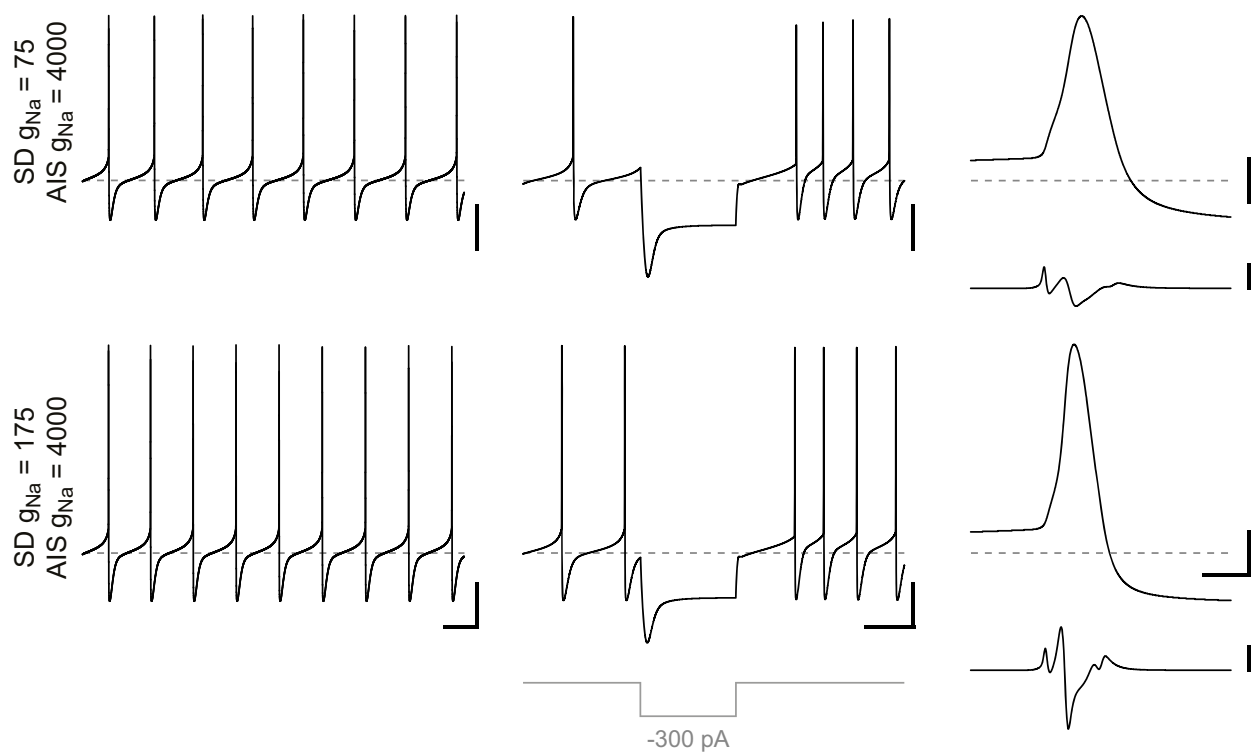

Figure 7. Validation of the average-morphology model. $A$, Left, Schematic representation of the average-morphology model depicting all the neuronal compartments included in the simulation. Right, Table indicating the length and diameters of the model compartments. While the axon, axon start (Ax-st) and the soma were modeled as cylinders (constant diameter), all other compartments were modeled as truncated cones. ABD, ax-st, and AIS length and diameters were based on measurements performed on confocal reconstructions of all the neurons included in the study. $\boldsymbol{B}$, Left, Line and scatter plot representing the relationship between AIS $\mathrm{g}_{\mathrm{Na}}$ and the amplitude of the IS $\mathrm{d}^{2} \mathrm{v} / \mathrm{dt}^{2}$ component recorded at the soma. Right, Line and scatter plot representing the relationship between $S D g_{\mathrm{Na}}$ and the amplitude of the $S D \mathrm{~d}^{2} \mathrm{v} / \mathrm{dt}^{2}$ component recorded at the soma. The data from 56 simulations (combinations of $50-200 \mathrm{pS} / \mu \mathrm{m}^{2} \mathrm{SD} \mathrm{g}_{\mathrm{Na}}$ and $1000-8000 \mathrm{pS} / \mu \mathrm{m}^{2} \mathrm{AIS} \mathrm{g}_{\mathrm{Na}}$ ) are represented and show the strict dependence of the two phases of the AP on the respective densities of sodium conductance in the AIS and the SD compartments. C, Voltage traces representing the pacemaker activity (left), the response to a hyperpolarizing current step (middle, $-300 \mathrm{pA}$ ), and the waveform of the somatic AP and its second time-derivative (right) in the average-morphology model with a low density of SD $\mathrm{g}_{\mathrm{Na}}\left(75 \mathrm{pS} / \mu \mathrm{m}^{2}\right.$, top traces) and a higher density of SD gNa (175 pS/ $\mu \mathrm{m}^{2}$, bottom traces). Calibration: C, Left, Middle, $20 \mathrm{mV}$ vertical, $200 \mathrm{~ms}$ horizontal; Right, $20 \mathrm{mV}$ vertical, $2 \mathrm{~ms}$ horizontal (top), $200 \mathrm{mV} \cdot \mathrm{ms}^{-2}$ vertical (bottom). Horizontal gray dotted lines indicate $-60 \mathrm{mV}$ (voltage traces).

quency by as much as $17.5 \%$ (Fig. $9 B$ ), the increase was reduced to $<8 \%$ for the average-morphology model ( 3 aDs; Fig. 10E) and to $<6 \%$ for the model with the longest soma-AIS distance ( $5 \mathrm{aDs}$; Fig. 10F). Overall, these results reinforce the conclusions obtained with the variations of $\mathrm{g}_{\mathrm{Na}}$ (Fig. 8), as they demonstrate that morphological variations of the SD compartment have a stronger influence on pacemaking frequency than changes of the AIS: while SD variations can modulate pacemaking frequency by as much as $109 \%$, frequency changes due to AIS manipulations do not exceed $18 \%$. 
A

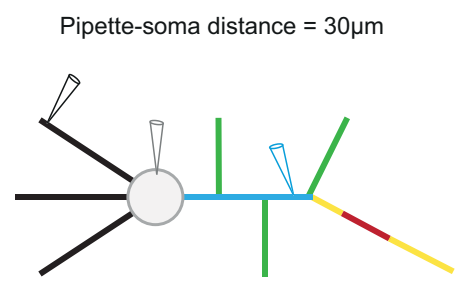

C

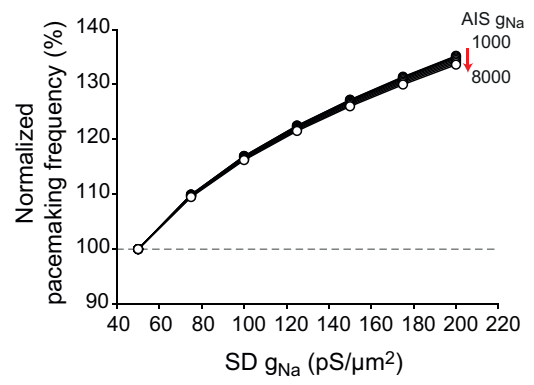

B

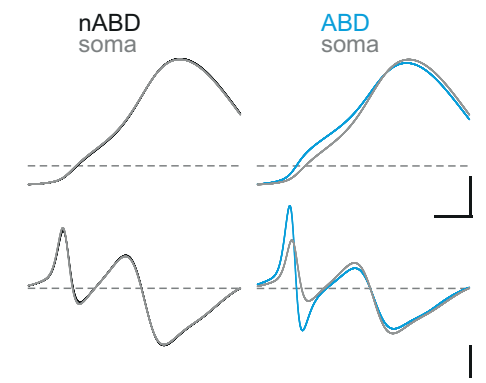

D
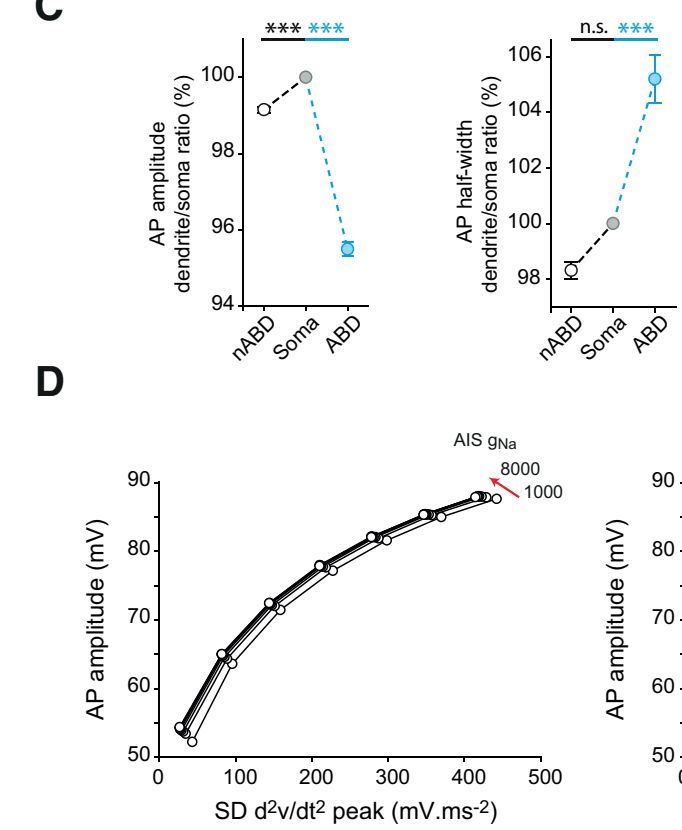

$\mathbf{F}$

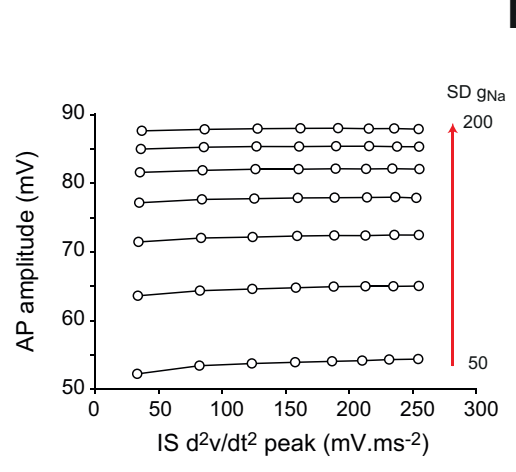

E

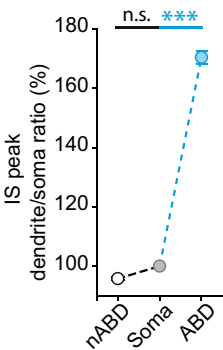

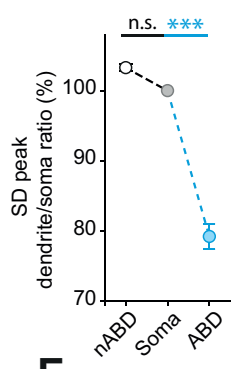

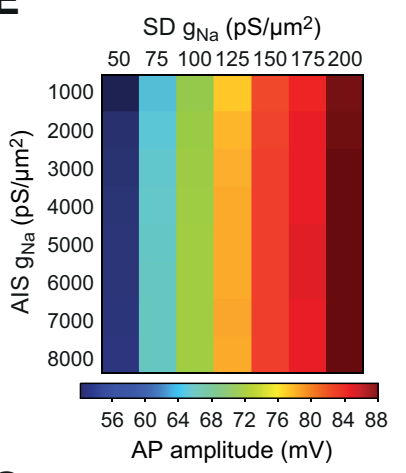

G

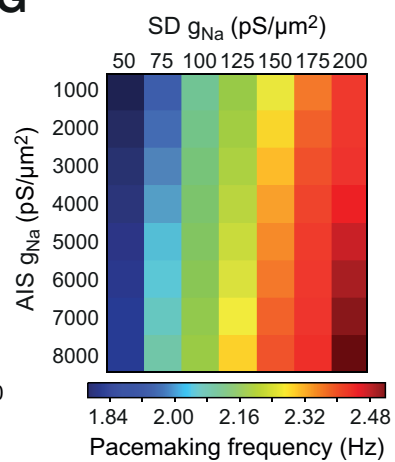

Figure 8. Multicompartment modeling of somatodendritic and AIS $g_{\mathrm{Na}}$ variations. $A$, Schematic representation of the average-morphology model with the location of the simulated recordings. $B$, Voltage traces representing the voltage waveform (top) and the secondary derivative of voltage (bottom) for an AP back-propagating in the ABD, the soma, and the nABD in the $75 / 4000 \mathrm{pS} / \mu \mathrm{m}^{2}$ model. C, Line and scatter plots representing the changes (from left to right) in AP amplitude, AP half-width, IS $\mathrm{d}^{2} \mathrm{v} / \mathrm{dt}^{2}$, and SD d $\mathrm{d}^{2} \mathrm{v} / \mathrm{dt}^{2}$ components during the back-propagation. The data from 9 models (combinations of $50-100 \mathrm{pS} / \mu \mathrm{m}^{2} \mathrm{SD} g_{\mathrm{Na}}$ and $3000-5000 \mathrm{pS} / \mu \mathrm{m}^{2} \mathrm{AIS} \mathrm{g}_{\mathrm{Na}}$ ) were pooled and averaged. $\boldsymbol{D}$, Line and scatter plots representing the relationship between the amplitudes of the SD d $\mathrm{d}^{2} \mathrm{v} / \mathrm{dt}^{2}$ component (left) or the IS $\mathrm{d}^{2} \mathrm{v} / \mathrm{dt}^{2}$ component (right) and AP amplitude in the model. $\boldsymbol{E}$, Heatmap representation of the change in AP amplitude as a function of SD and AIS $g_{\mathrm{Na}}$. The data from 56 simulations (combinations of $50-200 \mathrm{pS} / \mu \mathrm{m}^{2} S D g_{\mathrm{Na}}$ and $1000-8000 \mathrm{pS} / \mu \mathrm{m}^{2} \mathrm{AIS} \mathrm{g}_{\mathrm{Na}}$ ) are shown. $\boldsymbol{F}$, Line and scatter plots representing the relationship between $S D g_{\mathrm{Na}}$ (left) or AIS $g_{\mathrm{Na}}$ (right) and pacemaking frequency in the 56 models presented in $\boldsymbol{D}$. $\mathbf{G}$, Heatmap representation of the change in pacemaking frequency as a function of $S D$ and AIS $g_{\mathrm{Na}}$. The data from the same 56 simulations presented in $\boldsymbol{D}-\boldsymbol{F}$ are shown. Horizontal dotted lines indicate $-40 \mathrm{mV}(\boldsymbol{B}$, top $)$ and $0 \mathrm{mV} \cdot \mathrm{ms}^{-2}$ (B, bottom). $\boldsymbol{C}$, Normalized data are represented as mean $\pm S E M$. ${ }^{* * *} p<0.001$, n.s. not statistically significant. Calibration: $\boldsymbol{B}, 20 \mathrm{mV}$ vertical, $0.5 \mathrm{~ms}$ horizontal (top); $100 \mathrm{mV} \cdot \mathrm{ms}^{-2}$ vertical, $0.5 \mathrm{~ms}$ horizontal (bottom). Horizontal gray dotted lines indicate $-40 \mathrm{mV}(\boldsymbol{B}$, top) and 0 $\mathrm{mV} \cdot \mathrm{ms}^{-2}(\boldsymbol{B}$, bottom).

\section{Simulating realistic cell-to-cell variations in morphology}

The results from the multicompartment modeling presented so far demonstrate a strong role of the SD compartment and a weaker role of the AIS in the control of AP shape and pacemaking frequency. However, our experimental measurements failed to show clear relationships between morphology and electrophysiological output (see Fig. 4). This difference might be explained by several facts: (1) in the modeling approach, a few parameters (one to three) are changed at a time, and all other variables are kept constant. This may unveil weak relationships obscured in real 
A

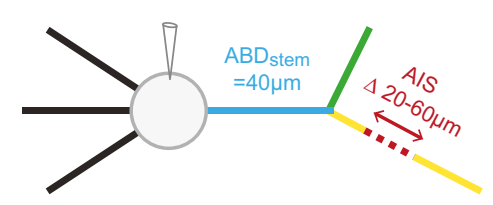

B
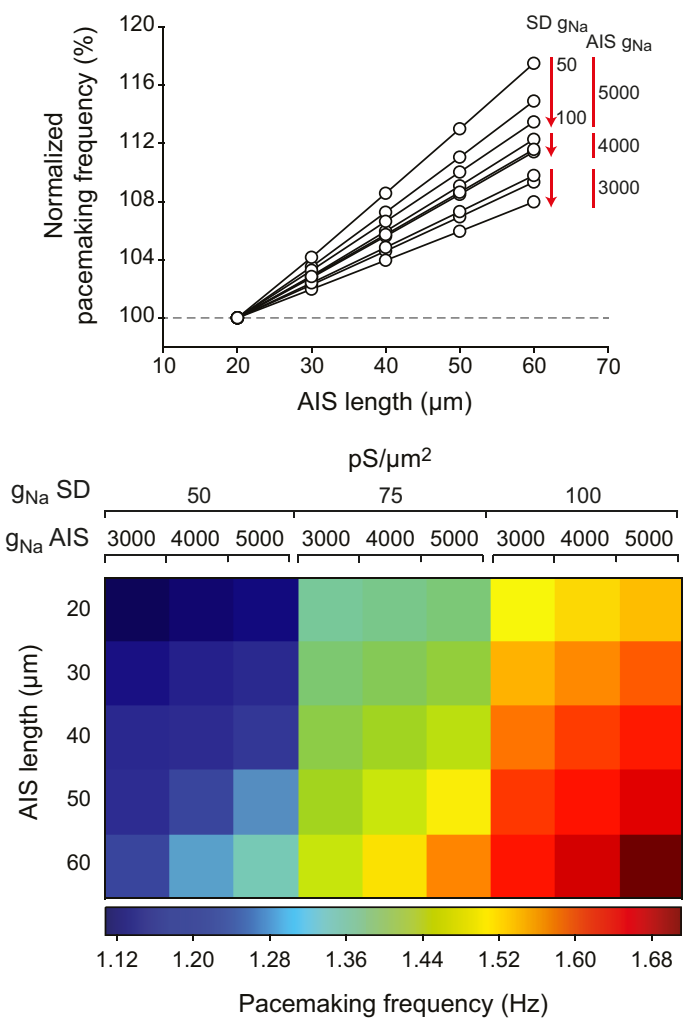

C

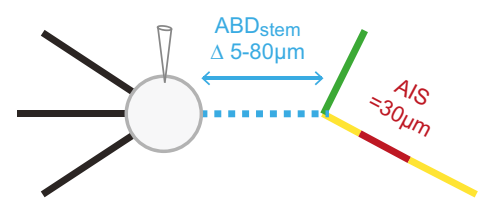

D
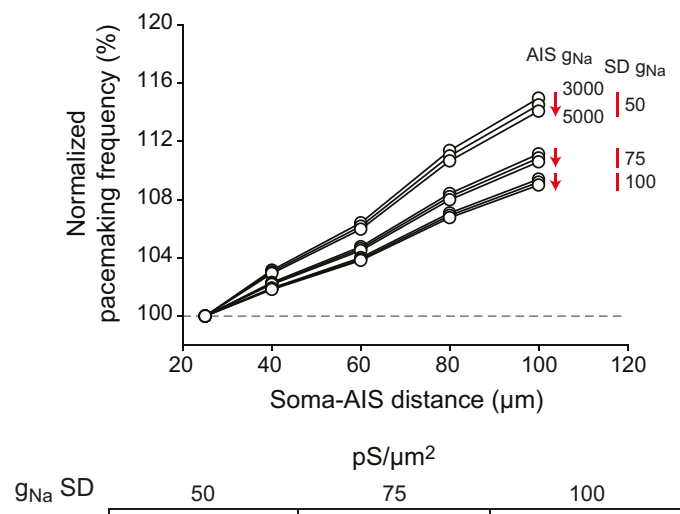

$g_{\mathrm{Na}}$ AIS $3000 \quad 4000 \quad 5000 \quad 3000 \quad 4000 \quad 5000 \quad 3000 \quad 4000 \quad 5000$

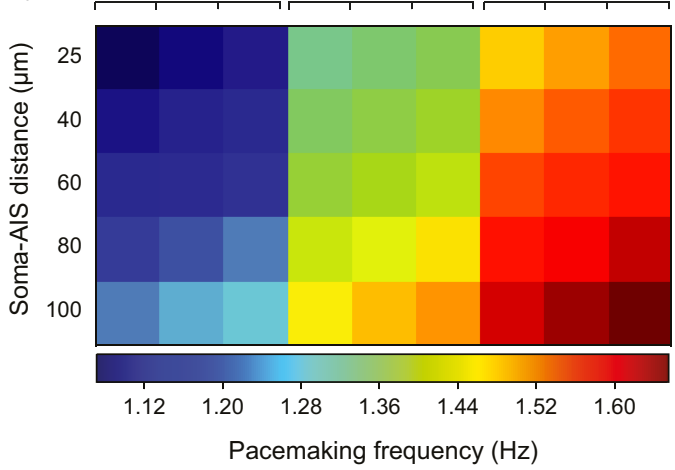

Figure 9. Multicompartment modeling of AIS and ABD morphological variations. $\boldsymbol{A}$, Schematic representation of the variations in AIS length simulated in the model. $\boldsymbol{B}$, Top, Line and scatter plot representing the effect of changing AIS length on pacemaking frequency. The data from 9 simulations (combinations of $50-100 \mathrm{pS} / \mu \mathrm{m}^{2} \mathrm{SD} \mathrm{g}_{\mathrm{Na}}$ and $3000-5000 \mathrm{pS} / \mu \mathrm{m}^{2} \mathrm{AIS} \mathrm{g}_{\mathrm{Na}}$ ) are represented. Bottom, Heatmap representation of the effect of changing AIS length (vertical axis) and SD $g_{\mathrm{Na}}$ and AIS $g_{\mathrm{Na}}$ (horizontal axis) on pacemaking frequency (color coding). $C$, Schematic representation of the variations in soma-AIS distance simulated in the model. $\boldsymbol{D}$, Top, Line and scatter plot representing the effect of changing soma-AIS distance on pacemaking frequency for the 9 combinations already presented in $\boldsymbol{A}-\boldsymbol{C}$. Bottom, Heatmap representation of the effect of changing soma-AIS distance (vertical axis) and SD $g_{\mathrm{Na}}$ and AIS $g_{\mathrm{Na}}$ (horizontal axis) on pacemaking frequency (color coding).

neurons by the biological variability of other morphological parameters (length, diameters of compartments, etc.); (2) the complexity of the dendritic compartment was greatly simplified in our model, and the topology of this compartment proved to be essential for pacemaking control (Fig. 10); and (3) as shown in Figure 2, many morphological parameters change in a not-socoordinated manner from neuron to neuron: $\mathrm{ABD}_{\text {stem }}$ length and the number of aDs are not strictly correlated, AIS length and soma-AIS distance are not correlated, etc.

To test whether the complexity of cell-to-cell morphological variations could explain the lack of relationship between electrophysiological output and morphology, we decided to simulate the firing behavior of the 37 neurons where full reconstruction was achieved (Fig. 11). To be as close as possible to the real morphology of the neurons, experimentally measured values were used for soma volume and diameters of primary ABD and nABDs, aDs, axon, and AIS. All compartment lengths, except axon length (set to $800 \mu \mathrm{m}$ ), were also taken from measurements. Three examples of these "real morphology" models are presented in Figure 11A. On these 37 morphologies, we simulated the 9 combinations of SD and AIS $\mathrm{g}_{\mathrm{Na}}$ already used in Figures 8, 9, and
10, obtaining 333 models based on real morphology. In these conditions, we replicated the correlation between $S D \mathrm{~d}^{2} \mathrm{v} / \mathrm{dt}^{2}$ and AP amplitude and the absence of correlation between IS $\mathrm{d}^{2} \mathrm{v} / \mathrm{dt}^{2}$ and AP amplitude observed in our experimental recordings (Fig. $11 B$ vs Fig. $3 E$ ). Moreover, the range of AP amplitudes was very similar to the one measured in the real neurons $(40-90 \mathrm{mV}$ vs $48-78 \mathrm{mV}$; Fig. $11 \mathrm{~B}$ vs Fig. $3 D$ ). We then tested whether we could detect significant statistical dependences between morphological parameters and pacemaking frequency. Although the frequencies were slightly faster than the ones observed in real neurons, the variability range obtained was very similar (5-fold, from 1.18 to 6 $\mathrm{Hz}$; Fig. $11 \mathrm{C}$ vs 5 -fold, from 0.59 to $3.24 \mathrm{~Hz}$; Fig. $4 C$ ). In addition, consistent with our experimental results, neither AIS length nor soma-AIS distance correlated with pacemaking frequency (Fig. $11 C)$. Interestingly, though, while increasing $\mathrm{SD} \mathrm{g}_{\mathrm{Na}}$ from 50 to $100 \mathrm{pS} / \mu \mathrm{m}^{2}$ in these real-morphology models produced an average increase in frequency of $23 \%$ (from 3.66 to $4.49 \mathrm{~Hz}$ ), increasing AIS $\mathrm{g}_{\mathrm{Na}}$ from 3000 to $5000 \mathrm{pS} / \mu \mathrm{m}^{2}$ only produced a $2 \%$ increase in frequency (from 4.1 to $4.19 \mathrm{~Hz}$; Fig. 11C). Moreover, the sensitivity to changes in $\mathrm{g}_{\mathrm{Na}}$ density was much stronger for model neurons firing at low frequencies than for neurons firing at 
A

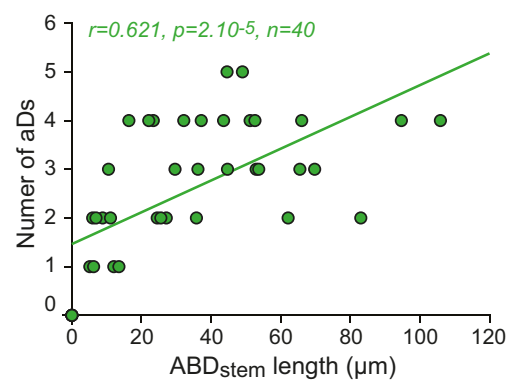

C

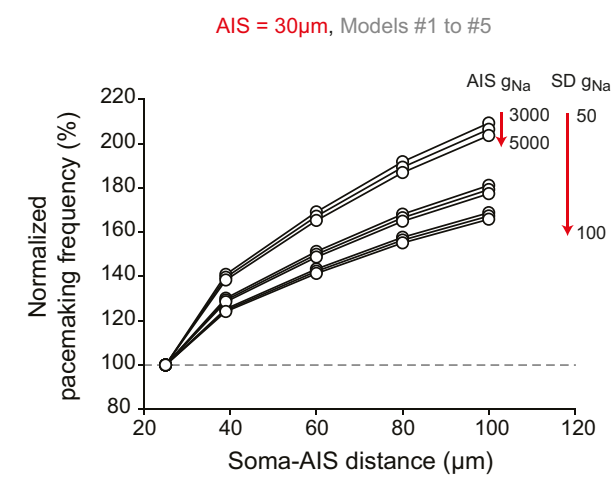

$\mathbf{E}$

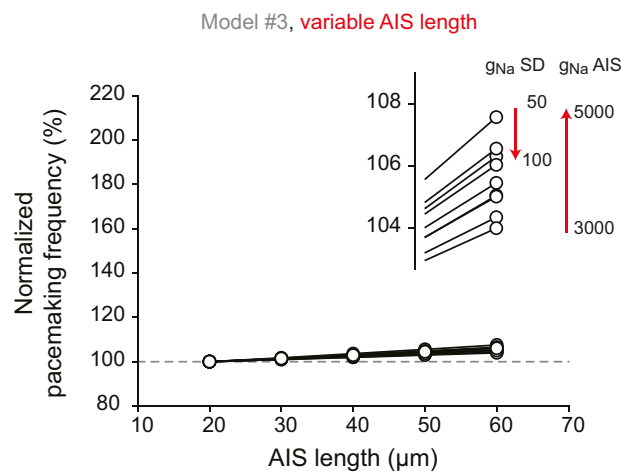

B

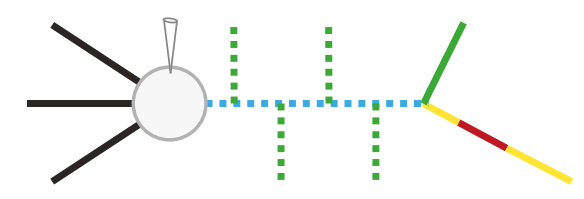

\begin{tabular}{|l|c|c|c|c|c|}
\hline Model \# & 1 & 2 & 3 & 4 & 5 \\
\hline ABD stem $(\mu \mathrm{m})$ & 5 & 20 & 40 & 60 & 80 \\
soma-AIS $(\mu \mathrm{m})$ & 25 & 40 & 60 & 80 & 100 \\
aDs & 1 & 2 & 3 & 4 & 5 \\
\hline
\end{tabular}

D

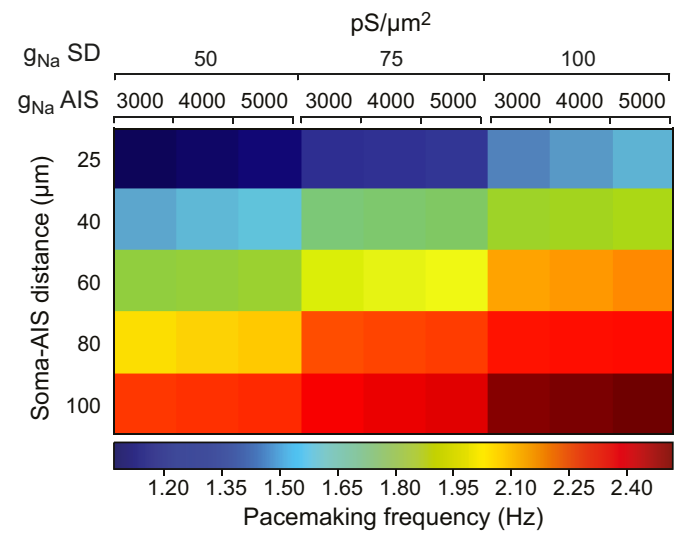

Model \#5, variable AIS length

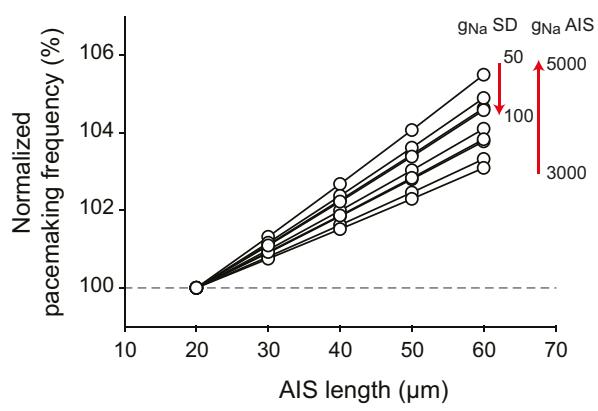

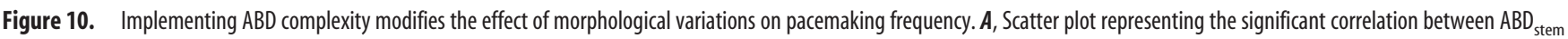
length and number of aDs observed in the 40 reconstructed SNc DA neurons. $\boldsymbol{B}$, Schematic representation of the variations in soma-AIS distance and the associated variation in number of aDs applied to the multicompartment model. $C$, Line and scatter plot representing the effect of changing soma-AIS distance on the pacemaking frequency in the model. The 9 combinations of $S D g_{\mathrm{Na}}$ and AIS $g_{\mathrm{Na}}$ already presented in Figure 9 were used. $D$, Heatmap representation of the raw values of pacemaking frequency depending on $S D g_{\mathrm{Na}^{\prime}} A I S g_{\mathrm{Na}}$ (horizontal axis), and soma-AIS distance (vertical axis). $E$, Line and scatter plot representing the effect of changing AIS length on pacemaking frequency in the average-morphology model (soma-AIS $=60 \mu \mathrm{m}, 3$ aDs). Inset, Expanded view of the points for AIS length $=60 \mu \mathrm{m} . \boldsymbol{F}$, Line and scatter plot representing the effect of changing AIS length on pacemaking frequency in the model with $5 \mathrm{aDs}$ (soma-AIS $=100 \mu \mathrm{m}$ ).

high frequencies, with increases in frequency reaching $140 \%$ and $31 \%$ for variations in SD an AIS $\mathrm{g}_{\mathrm{Na}}$, respectively (Fig. 12).

In conclusion, the multicompartment modeling of $\mathrm{SNc}$ DA neurons demonstrated that the SD compartment plays a major role in controlling both AP shape and pacemaking frequency, while the AIS has a much weaker influence on these parameters. In addition, the simulation of real morphologies suggests that the complexity of cellto-cell morphological variations masks the respective influence of these compartments on electrophysiological output.

\section{Discussion}

While many studies have reported an essential role of AIS geometry in excitability in different neuronal types (Kuba et al., 2006, 2014; Grubb and Burrone, 2010; Kuba, 2012; Gutzmann et al., 2014; Chand et al., 2015; Hamada et al., 2016; Meza et al., 2018), the current work underlines the weak role played by the AIS in the tuning of activity of rat SNc DA neurons: both AP characteristics and pacemaking frequency appear insensitive to variations in AIS morphological or biophysical properties. Multicompartment modeling constrained by experimental recordings of sodium, delayed rectifier potassium currents and AP back-propagation shed light on this robustness to morphological variations: while the excitability of the somatodendritic compartment outweighs the influence of the AIS on neuronal output, its complex morphological variations from neuron to neuron prevent the extraction of simple rules relating morphology to pattern of activity. The robustness of firing of SNc DA neurons to large morphological variations may be part of their distinctive physiological profile. 
A
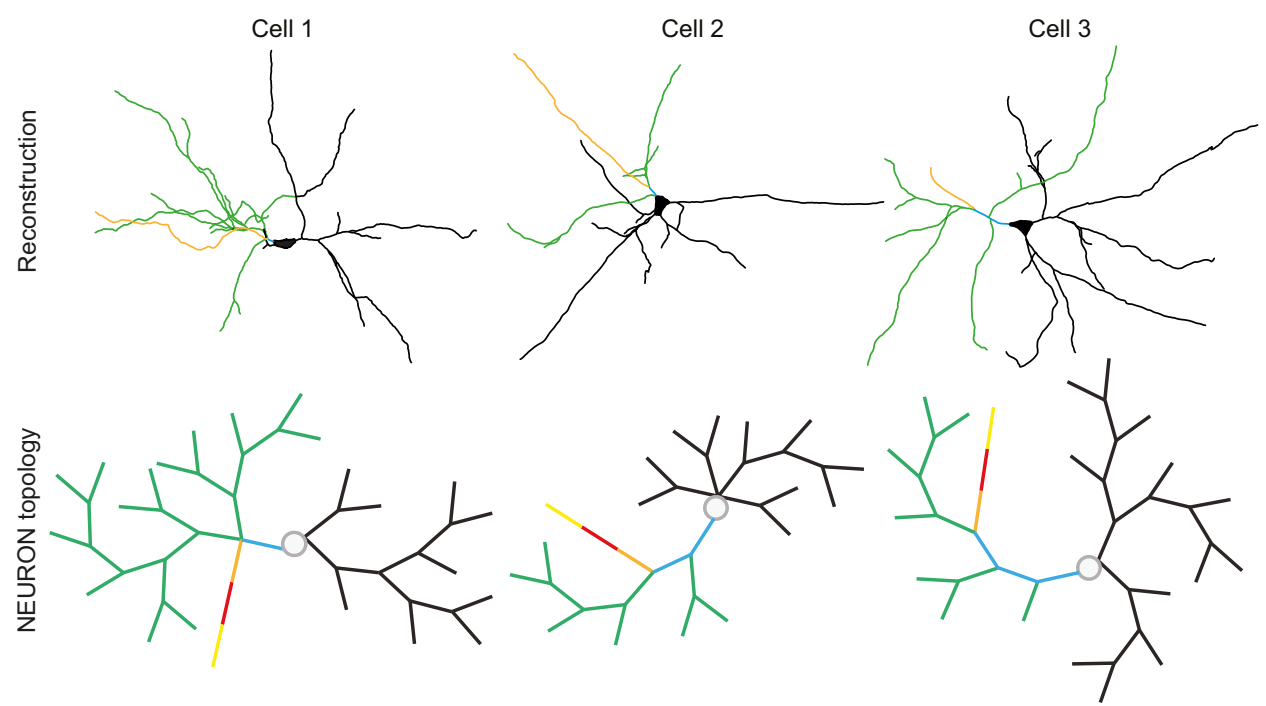

\begin{tabular}{|c|c|c|c|c|c|c|c|}
\hline & $\begin{array}{c}\text { number of } \\
\text { Iary }_{\text {nABDs }}\end{array}$ & $\begin{array}{c}\text { Soma volume } \\
\left(\mu \mathrm{m}^{3}\right)\end{array}$ & $\begin{array}{c}\text { ABD length } \\
(\mu \mathrm{m})\end{array}$ & $\begin{array}{c}\text { number of } \\
\mathrm{aDs}\end{array}$ & $\begin{array}{c}\text { Ax-st length } \\
(\mu \mathrm{m})\end{array}$ & $\begin{array}{c}\text { AIS length } \\
(\mu \mathrm{m})\end{array}$ & $\begin{array}{c}\text { Axon length } \\
(\mu \mathrm{m})\end{array}$ \\
\hline Cell 1 & 2 & 1503 & 11 & 2 & 24 & 27 & 800 \\
\hline Cell 2 & 4 & 3180 & 25 & 2 & 19 & 36 & 800 \\
\hline Cell 3 & 2 & 3115 & 70 & 3 & 21 & 25 & 800 \\
\hline
\end{tabular}

B
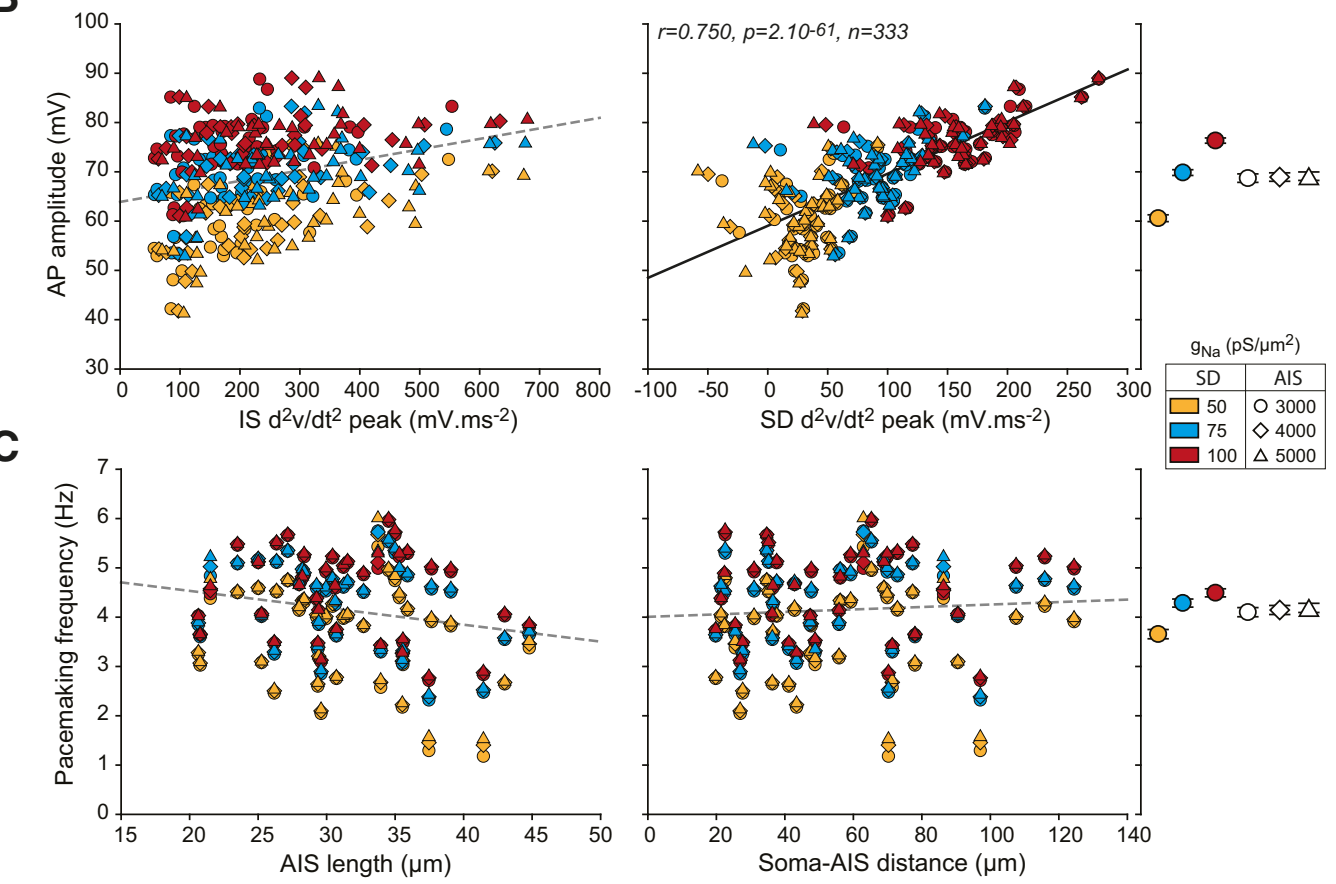

Figure 11. Simulating electrophysiological output using realistic morphologies. $A$, Skeleton (top) and topological representations from the NEURON software (middle) for 3 cells representative of the morphological diversity across our 37 neuron sample. Bottom, Values of the main morphological parameters for the 3 cells presented above. $\boldsymbol{B}$, Scatter plots representing the relationship between IS $\mathrm{d}^{2} \mathrm{v} / \mathrm{dt}^{2}$ peak (left) or SD $\mathrm{d}^{2} \mathrm{v} / \mathrm{dt}^{2}$ peak (right) and AP amplitude in the 37 real-morphology models. The data from 9 simulations (combinations of $50-100 \mathrm{pS} / \mu \mathrm{m}^{2} \mathrm{SD} \mathrm{g}_{\mathrm{Na}}$ and $3000-5000 \mathrm{pS} / \mu \mathrm{m}^{2}$ AIS $g_{\mathrm{Na}}$, distinguished by colors and symbols) are represented $\left(37 \times 9=333\right.$ points). Far right, The average AP amplitude \pm SEM for each SD $g_{\mathrm{Na}}$ density and each AIS $g_{\mathrm{Na}}$ density is represented. C, Scatter plots representing the lack of relationship between AIS length (left) or soma-AIS distance (right) and pacemaking frequency in the 333 real-morphology simulations. Far right, The average pacemaking frequency $\pm S E$ for each $S D g_{\mathrm{Na}}$ density and each $A I S g_{\mathrm{Na}}$ density is represented. Plain lines indicate significant correlations between parameters. $r, p$, and $n$ values are given on the corresponding plots. Dotted lines indicate nonsignificant correlations.

\section{Morphological and biophysical constraints of neuronal output in SNc DA neurons}

Several theoretical studies investigated the impact of dendritic morphology on AP back-propagation and neuronal output (Mainen and Sejnowski, 1996; Mainen et al., 1996; Vetter et al., 2001; van Ooyen et al., 2002; Weaver and Wearne, 2008; Hay et al., 2013; Gulledge and Bravo, 2016). One study in particular
(Vetter et al., 2001) suggested that the relative simplicity of SNc DA neuron dendrites is optimal for both back- and forwardpropagation of APs, whereas the complex dendrites of pyramidal and Purkinje neurons are unfavorable to AP back-propagation. The efficiency of AP back-propagation is indeed predicted from the number of dendritic branching points and the associated impedance mismatches. In this perspective, the presence of a signif- 
icant number of aDs in between the spike initiation site (the AIS) and the soma observed in SNc DA neurons should impair AP back-propagation. Yet, consistent with previous observations (Häusser et al., 1995; Gentet and Williams, 2007), faithful AP back-propagation during spontaneous pacemaking was present in all our current-clamp recordings, independent of ABD complexity and somaAIS distance.

We also observed faithful AP backpropagation and regular pacemaking in nearly all versions of our multicompartment model, which predicted that aDs should have a strong positive influence on pacemaking frequency. As modeling results are inherently dependent on the morphology and conductance densities chosen for each compartment, it is important to note that we used realistic morphologies coming from full neuronal reconstructions and conductance densities measured in outside-out dendritic recordings. Interestingly, the measured average density for $\mathrm{g}_{\mathrm{Na}}\left(75 \mathrm{pS} / \mu \mathrm{m}^{2}\right)$ is close to the values reported in other faithfully back-propagating neuronal types $\left(90-110 \mathrm{pS} / \mu \mathrm{m}^{2}\right.$ ) (Bischofberger and Jonas, 1997; Martina et al., 2000). Consistently, Vetter et al. (2001) showed that full AP back- and forward-propagation is achieved in SNc DA neuron models with SD $\mathrm{g}_{\mathrm{Na}}$ of 40 and $80 \mathrm{pS} / \mu \mathrm{m}^{2}$, respectively. Our measurements of $\mathrm{SD} \mathrm{g}_{\mathrm{KDR}}$ and the resulting $\mathrm{g}_{\mathrm{Na}} / \mathrm{g}_{\mathrm{KDR}}(0.5)$ also match the low sensitivity of SNc DA neuron to potassium conductance predicted using modeling (half-maximal back-propagation for $\mathrm{g}_{\mathrm{Na}} / \mathrm{g}_{\mathrm{KDR}}=0.64$ ) (Vetter et al., 2001). Associated with AIS conductance densities that produce the appropriate amplitude for the IS component of the AP, these realistic $\mathrm{SD}$ conductance densities not only quantitatively reproduce the AP back-propagation profile but also turn out to reveal an essential role of the SD compartment in setting pacemaking frequency.

\section{Pacemaking and the localization of coupled oscillators}

Although the debate is still ongoing (Chan et al., 2007; Puopolo et al., 2007; Guzman et al., 2009; Drion et al., 2011), the Cav1.3 calcium and Nav1 sodium channels seem to be the main components of the oscillators giving rise to pacemaking. Whether the soma-, dendrite-, or axon-located channels prevail in this process has also been investigated (Wilson and Callaway, 2000; Kuznetsova et al., 2010; Tucker et al., 2012; Jang et al., 2014; Meza et al., 2018). In particular, focal blockade of sodium channels (Jang et al., 2014) and dynamic-clamp injection of sodium conductances (Tucker et al., 2012) highlighted the importance of sodium channels located in the soma and proximal dendrites in the control of pacemaking frequency. Interestingly, a recent study performed in the mouse suggested that AIS-located sodium channels may play an essential role in setting the pacemaking frequency of SNc DA neurons in vivo (Meza et al., 2018). Our experimental results contrast with this latter observation, and our model predicts that variations in AIS $\mathrm{g}_{\mathrm{Na}}$ or AIS length have a minor influence on pacemaking frequency compared with changes in $\mathrm{SD} \mathrm{g}_{\mathrm{Na}}$ or $\mathrm{ABD}_{\text {stem }}$ length. As an example, the addition of $\mathrm{aDs}$ on the $\mathrm{ABD}$ induces a strong increase in pacemaking frequency while minimizing the influence of variations in AIS morphology (Fig.
10). Our results are consistent with the theory of coupled oscillators where the respective surface areas of the neuronal compartments containing the different oscillators essentially determine their impact on the final pacemaking frequency (Wilson and Callaway, 2000).

In this perspective, the results obtained by Meza et al. (2018) are rather surprising. We cannot rule out interspecies or experimental (in vitro vs anesthetized) differences to explain the contrast between our results and their results. However, the influence of AIS geometry on pacemaking frequency reported by Meza et al. (2018) can be at least partly explained by the low SD $g_{\mathrm{Na}}$ density used in their model $\left(5 \mathrm{pS} / \mu^{2} \mathrm{~m}^{2}, 15\right.$ times less than the density measured in our recordings), which would inevitably lead to an underestimation of the influence of the SD compartment and a correlated overestimation of the influence of the AIS on pacemaking.

\section{Robustness of SNc DA neuron output to morphological variations}

While manipulating each morphological or biophysical parameter on an average morphology or simplified model allowed us to demonstrate the predominant influence of the $\mathrm{ABD}$ complexity and excitability on pacemaking frequency, our experimental observations failed to show any significant correlation between morphological parameters and neuronal output (AP properties or pacemaking frequency). This may seem paradoxical, but modeling the "real" morphology of reconstructed neurons is shedding light on this robustness to morphological variation. As already discussed, while the AP is always initiated in the AIS, the contribution of this compartment to pacemaking is small compared with the weight of the SD compartment. AP shape is thus mainly determined by the contribution of SD sodium currents, which ensure the amplification of the AIS-initiated AP and its full backpropagation. Together, this may explain why SNc DA neurons tolerate so much variation in AIS geometry compared with other neuronal types (Kuba et al., 2006, 2014; Grubb and Burrone, 2010). This situation is reminiscent of the observation made in 
hippocampal oriens-alveus interneurons (Martina et al., 2000), where faithful AP back-propagation due to a high density of somatodendritic sodium channels occurs in parallel with large variations in soma-AIS distance (up to $110 \mu \mathrm{m}$ ).

On the other hand, we did not find clear relationships between dendritic morphology and spontaneous firing. Several theoretical studies showed that morphological changes of the dendritic arborization may strongly modify excitability or even change the pattern of activity of model neurons (Mainen and Sejnowski, 1996; van Ooyen et al., 2002; Weaver and Wearne, 2008). Our realistic morphology models reproduce the lack of correlation between morphological measurements and pacemaking frequency, suggesting that too many morphological and biophysical parameters are varying from neuron to neuron (ABD diameter and length, number of aDs, soma volume, $\mathrm{SD} \mathrm{g}_{\mathrm{Na}}$ ) to extract a simple rule linking morphology to excitability in these neurons. While we tried to base our realistic modeling only on morphological measurements, very fine diameters $(<0.5 \mu \mathrm{m})$ could not be accurately measured using a confocal microscope. Therefore, we assumed a regular tapering for secondary and higher-order dendrites, reaching a final diameter of $0.5 \mu \mathrm{m}$. Simulating the whole range of SD and AIS $\mathrm{g}_{\mathrm{Na}}$ densities on each reconstructed neuron allowed us to estimate the respective impact of cell-to-cell variations in morphology and biophysics on pacemaking frequency. While varying $S D \mathrm{~g}_{\mathrm{Na}}$ over its whole biological range in a constant morphology can increase frequency by as much as $100 \%$ (data not shown), a much larger range of frequencies is obtained for a constant $\mathrm{g}_{\mathrm{Na}}$ using the range of morphological variations (up to $500 \%$ increase; Fig. $11 C$ ). Therefore, the model predicts that, in biological conditions, SD morphological variations are responsible for most of the observed cell-to-cell variation in pacemaking frequency. In this respect, the approximation we had to make about the regular tapering of secondary dendrites likely led us to underestimate the variations in pacemaking frequency due to cell-to-cell variations in morphology. Reminiscent of recent observations made on crustacean stomatogastric nervous system neurons (Otopalik et al., 2017a,b), our results therefore suggest that $\mathrm{SNc}$ DA neurons may not comply with morphological optimization principles (Chklovskii, 2000; Cuntz et al., 2007) and that the strong $\mathrm{SD} \mathrm{g}_{\mathrm{Na}}$ density may be partly responsible for this tolerance to morphological variation.

\section{Physiological underpinnings of the robustness to morphological variation}

Most of the studies investigating the influence of AIS geometry on neuronal excitability have been performed on cell types that are activated in response to spatially and temporally ordered synaptic inputs (Kuba et al., 2006, 2014; Meeks and Mennerick, 2007; Kress et al., 2008, 2010; Fried et al., 2009; Grubb and Burrone, 2010; Kuba, 2012; Scott et al., 2014; Thome et al., 2014). These neurons follow the law of dynamic polarization proposed by Cajal (1952), where information propagates from the dendrites to the soma, and then to the axon, this organization being optimal for synaptic integration-mediated spike firing. SNc DA neurons differ significantly from these neuronal types both from a functional and morphological point of view. First, SNc DA neurons are spontaneously active: their pacemaking pattern of activity is observed both in vitro and in vivo; even though in the latter case, it is modulated by synaptic inputs (Gantz et al., 2018). Second, these neurons are not only releasing DA from their axonal terminals in the striatum but also communicate locally in the $\mathrm{SNc}$ via AP-dependent somatodendritic release of DA (Vandecasteele et al., 2008; Yee et al., 2019). These specificities explain why soma- todendritic excitability (via sodium and calcium channels) may be so central to SNc DA neurons, as it supports both the voltage oscillations giving rise to pacemaking activity and the faithful AP back-propagation necessary for somatodendritic release of DA. The hypothesis we are proposing is that, once these conditions are fulfilled, AIS geometry has a minor impact on SNc DA neuron excitability, such that large variations in soma-AIS distance or AIS length are tolerated. Additionally, under the constraint of faithful back-propagation, keeping the spike initiation site away from the voltage-sink effect produced by the soma might actually be beneficial (Hesse and Schreiber, 2015; Niven, 2015; Kole and Brette, 2018).

\section{References}

Amendola J, Woodhouse A, Martin-Eauclaire MF, Goaillard JM (2012) $\mathrm{Ca}(2)(+) /$ cAMP-sensitive covariation of $\mathrm{I}(\mathrm{A})$ and $\mathrm{I}(\mathrm{H})$ voltage dependences tunes rebound firing in dopaminergic neurons. J Neurosci $32: 2166-2181$

Bean BP (2007) The action potential in mammalian central neurons. Nat Rev Neurosci 8:451-465.

Bischofberger J, Jonas P (1997) Action potential propagation into the presynaptic dendrites of rat mitral cells. J Physiol 504:359-365.

Blythe SN, Wokosin D, Atherton JF, Bevan MD (2009) Cellular mechanisms underlying burst firing in substantia nigra dopamine neurons. J Neurosci 29:15531-15541.

Cajal SR (1952) Histologie du systeme nerveux de l'homme and des vertebres: Consejo Superior de Investigaciones Científicas. Madrid: Instituto Ramón y Cajal.

Carnevale NT, Hines ML (2006) The NEURON book: Cambridge: Cambridge UP.

Chan CS, Guzman JN, Ilijic E, Mercer JN, Rick C, Tkatch T, Meredith GE, Surmeier DJ (2007) 'Rejuvenation' protects neurons in mouse models of Parkinson's disease. Nature 447:1081-1086.

Chand AN, Galliano E, Chesters RA, Grubb MS (2015) A distinct subtype of dopaminergic interneuron displays inverted structural plasticity at the axon initial segment. J Neurosci 35:1573-1590.

Chklovskii DB (2000) Optimal sizes of dendritic and axonal arbors in a topographic projection. J Neurophysiol 83:2113-2119.

Clark BD, Goldberg EM, Rudy B (2009) Electrogenic tuning of the axon initial segment. Neuroscientist 15:651-668.

Coombs JS, Curtis DR, Eccles JC (1957) The generation of impulses in motoneurones. J Physiol 139:232-249.

Cuntz H, Borst A, Segev I (2007) Optimization principles of dendritic structure. Theor Biol Med Model 4:21.

Debanne D, Campanac E, Bialowas A, Carlier E, Alcaraz G (2011) Axon physiology. Physiol Rev 91:555-602.

Destexhe A, Babloyantz A, Sejnowski TJ (1993) Ionic mechanisms for intrinsic slow oscillations in thalamic relay neurons. Biophys J 65: $1538-1552$

Ding S, Wei W, Zhou FM (2011a) Molecular and functional differences in voltage-activated sodium currents between GABA projection neurons and dopamine neurons in the substantia nigra. J Neurophysiol 106:30193034.

Ding S, Matta SG, Zhou FM (2011b) Kv3-like potassium channels are required for sustained high-frequency firing in basal ganglia output neurons. J Neurophysiol 105:554-570.

Drion G, Massotte L, Sepulchre R, Seutin V (2011) How modeling can reconcile apparently discrepant experimental results: the case of pacemaking in dopaminergic neurons. PLoS Comput Biol 7:e1002050.

Dufour MA, Woodhouse A, Amendola J, Goaillard JM (2014) Non-linear developmental trajectory of electrical phenotype in rat substantia nigra pars compacta dopaminergic neurons. Elife. Advance online publication. Retrieved October 20, 2014. doi:10.7554/eLife.04059.

Engel D, Seutin V (2015) High dendritic expression of Ih in the proximity of the axon origin controls the integrative properties of nigral dopamine neurons. J Physiol 593:4905-4922.

Fried SI, Lasker AC, Desai NJ, Eddington DK, Rizzo JF 3rd (2009) Axonal sodium-channel bands shape the response to electric stimulation in retinal ganglion cells. J Neurophysiol 101:1972-1987.

Gantz SC, Ford CP, Morikawa H, Williams JT (2018) The evolving under- 
standing of dopamine neurons in the substantia nigra and ventral tegmental area. Annu Rev Physiol 80:219-241.

Gentet LJ, Williams SR (2007) Dopamine gates action potential backpropagation in midbrain dopaminergic neurons. J Neurosci 27: 1892-1901.

González-Cabrera C, Meza R, Ulloa L, Merino-Sepúlveda P, Luco V, Sanhueza A, Oñate-Ponce A, Bolam JP, Henny P (2017) Characterization of the axon initial segment of mice substantia nigra dopaminergic neurons. J Comp Neurol 525:3529-3542.

Grace AA, Bunney BS (1983) Intracellular and extracellular electrophysiology of nigral dopaminergic neurons: 2. Action potential generating mechanisms and morphological correlates. Neuroscience 10:317-331.

Grace AA, Bunney BS (1984) The control of firing pattern in nigral dopamine neurons: single spike firing. J Neurosci 4:2866-2876.

Grace AA, Onn SP (1989) Morphology and electrophysiological properties of immunocytochemically identified rat dopamine neurons recorded in vitro. J Neurosci 9:3463-3481.

Grubb MS, Burrone J (2010) Activity-dependent relocation of the axon initial segment fine-tunes neuronal excitability. Nature 465:1070-1074.

Gulledge AT, Bravo JJ (2016) Neuron morphology influences axon initial segment plasticity. eNeuro 3:ENEURO.0085-15.2016.

Gutzmann A, Ergül N, Grossmann R, Schultz C, Wahle P, Engelhardt M (2014) A period of structural plasticity at the axon initial segment in developing visual cortex. Front Neuroanat 8:11.

Guzman JN, Sánchez-Padilla J, Chan CS, Surmeier DJ (2009) Robust pacemaking in substantia nigra dopaminergic neurons. J Neurosci 29: 11011-11019.

Hamada MS, Goethals S, de Vries SI, Brette R, Kole MH (2016) Covariation of axon initial segment location and dendritic tree normalizes the somatic action potential. Proc Natl Acad Sci U S A 113:14841-14846.

Häusser M, Stuart G, Racca C, Sakmann B (1995) Axonal initiation and active dendritic propagation of action potentials in substantia nigra neurons. Neuron 15:637-647.

Hay E, Schürmann F, Markram H, Segev I (2013) Preserving axosomatic spiking features despite diverse dendritic morphology. J Neurophysiol 109:2972-2981.

Hesse J, Schreiber S (2015) Externalization of neuronal somata as an evolutionary strategy for energy economization. Curr Biol 25:R324-R325.

Hines ML, Carnevale NT (1997) The NEURON simulation environment. Neural Comput 9:1179-1209.

Hines ML, Carnevale NT (2001) NEURON: a tool for neuroscientists. Neuroscientist 7:123-135.

Hu W, Tian C, Li T, Yang M, Hou H, Shu Y (2009) Distinct contributions of $\mathrm{Na}(\mathrm{v}) 1.6$ and $\mathrm{Na}(\mathrm{v}) 1.2$ in action potential initiation and backpropagation. Nat Neurosci 12:996-1002.

Jang J, Um KB, Jang M, Kim SH, Cho H, Chung S, Kim HJ, Park MK (2014) Balance between the proximal dendritic compartment and the soma determines spontaneous firing rate in midbrain dopamine neurons. J Physiol 592:2829-2844.

Kole MH, Brette R (2018) The electrical significance of axon location diversity. Curr Opin Neurobiol 51:52-59.

Kole MH, Stuart GJ (2012) Signal processing in the axon initial segment. Neuron 73:235-247.

Kole MH, Ilschner SU, Kampa BM, Williams SR, Ruben PC, Stuart GJ (2008) Action potential generation requires a high sodium channel density in the axon initial segment. Nat Neurosci 11:178-186.

Kress GJ, Dowling MJ, Meeks JP, Mennerick S (2008) High threshold, proximal initiation, and slow conduction velocity of action potentials in dentate granule neuron mossy fibers. J Neurophysiol 100:281-291.

Kress GJ, Dowling MJ, Eisenman LN, Mennerick S (2010) Axonal sodium channel distribution shapes the depolarized action potential threshold of dentate granule neurons. Hippocampus 20:558-571.

Kuba H (2012) Structural tuning and plasticity of the axon initial segment in auditory neurons. J Physiol 590:5571-5579.

Kuba H, Ishii TM, Ohmori H (2006) Axonal site of spike initiation enhances auditory coincidence detection. Nature 444:1069-1072.

Kuba H, Adachi R, Ohmori H (2014) Activity-dependent and activityindependent development of the axon initial segment. J Neurosci 34: 3443-3453.

Kuznetsova AY, Huertas MA, Kuznetsov AS, Paladini CA, Canavier CC (2010) Regulation of firing frequency in a computational model of a midbrain dopaminergic neuron. J Comput Neurosci 28:389-403.
Lezmy J, Lipinsky M, Khrapunsky Y, Patrich E, Shalom L, Peretz A, Fleidervish IA, Attali B (2017) M-current inhibition rapidly induces a unique CK2-dependent plasticity of the axon initial segment. Proc Natl Acad Sci U S A 114:E10234-E10243.

Lien CC, Jonas P (2003) Kv3 potassium conductance is necessary and kinetically optimized for high-frequency action potential generation in hippocampal interneurons. J Neurosci 23:2058-2068.

Liss B, Roeper J (2008) Individual dopamine midbrain neurons: functional diversity and flexibility in health and disease. Brain Res Rev 58:314-321.

Longair MH, Baker DA, Armstrong JD (2011) Simple neurite tracer: open source software for reconstruction, visualization and analysis of neuronal processes. Bioinformatics 27:2453-2454.

Lorincz A, Nusser Z (2008) Cell-type-dependent molecular composition of the axon initial segment. J Neurosci 28:14329-14340.

Lorincz A, Nusser Z (2010) Molecular identity of dendritic voltage-gated sodium channels. Science 328:906-909.

Mainen ZF, Sejnowski TJ (1996) Influence of dendritic structure on firing pattern in model neocortical neurons. Nature 382:363-366.

Mainen ZF, Carnevale NT, Zador AM, Claiborne BJ, Brown TH (1996) Electrotonic architecture of hippocampal CA1 pyramidal neurons based on three-dimensional reconstructions. J Neurophysiol 76:1904-1923.

Martina M, Vida I, Jonas P (2000) Distal initiation and active propagation of action potentials in interneuron dendrites. Science 287:295-300.

Matsuda W, Furuta T, Nakamura KC, Hioki H, Fujiyama F, Arai R, Kaneko T (2009) Single nigrostriatal dopaminergic neurons form widely spread and highly dense axonal arborizations in the neostriatum. J Neurosci 29:444-453.

Matsuda Y, Fujimura K, Yoshida S (1987) Two types of neurons in the substantia nigra pars compacta studied in a slice preparation. Neurosci Res 5:172-179.

Meeks JP, Mennerick S (2007) Action potential initiation and propagation in CA3 pyramidal axons. J Neurophysiol 97:3460-3472.

Meza RC, López-Jury L, Canavier CC, Henny P (2018) Role of the axon initial segment in the control of spontaneous frequency of nigral dopaminergic neurons in vivo. J Neurosci 38:733-744.

Migliore M, Cannia C, Canavier CC (2008) A modeling study suggesting a possible pharmacological target to mitigate the effects of ethanol on reward-related dopaminergic signaling. J Neurophysiol 99:2703-2707.

Niven JE (2015) Neural evolution: marginal gains through soma location. Curr Biol 25:R330-R332.

Otopalik AG, Sutton AC, Banghart M, Marder E (2017a) When complex neuronal structures may not matter. Elife 6:e23508.

Otopalik AG, Goeritz ML, Sutton AC, Brookings T, Guerini C, Marder E (2017b) Sloppy morphological tuning in identified neurons of the crustacean stomatogastric ganglion. Elife 6:e22352.

Palmer LM, Stuart GJ (2006) Site of action potential initiation in layer 5 pyramidal neurons. J Neurosci 26:1854-1863.

Platkiewicz J, Brette R (2010) A threshold equation for action potential initiation. PLoS Comput Biol 6:e1000850.

Prensa L, Parent A (2001) The nigrostriatal pathway in the rat: a single-axon study of the relationship between dorsal and ventral tier nigral neurons and the striosome/matrix striatal compartments. J Neurosci 21:72477260.

Puopolo M, Raviola E, Bean BP (2007) Roles of subthreshold calcium current and sodium current in spontaneous firing of mouse midbrain dopamine neurons. J Neurosci 27:645-656.

Putzier I, Kullmann PH, Horn JP, Levitan ES (2009) Dopamine neuron responses depend exponentially on pacemaker interval. J Neurophysiol 101:926-933.

Rasband MN (2010) The axon initial segment and the maintenance of neuronal polarity. Nat Rev Neurosci 11:552-562.

Rueden CT, Schindelin J, Hiner MC, DeZonia BE, Walter AE, Arena ET, Eliceiri KW (2017) ImageJ2: ImageJ for the next generation of scientific image data. BMC Bioinformatics 18:529.

Sakmann B, Neher E (1995) Single channel recordings. New York: Plenum. Schild JH, Khushalani S, Clark JW, Andresen MC, Kunze DL, Yang M (1993) An ionic current model for neurons in the rat medial nucleus tractus solitarii receiving sensory afferent input. J Physiol 469:341-363.

Schindelin J, Arganda-Carreras I, Frise E, Kaynig V, Longair M, Pietzsch T, Preibisch S, Rueden C, Saalfeld S, Schmid B, Tinevez JY, White DJ, Hartenstein V, Eliceiri K, Tomancak P, Cardona A (2012) Fiji: an opensource platform for biological-image analysis. Nat Methods 9:676-682. 
Schneider CA, Rasband WS, Eliceiri KW (2012) NIH image to ImageJ: 25 years of image analysis. Nat Methods 9:671-675.

Scott RS, Henneberger C, Padmashri R, Anders S, Jensen TP, Rusakov DA (2014) Neuronal adaptation involves rapid expansion of the action potential initiation site. Nat Commun 5:3817.

Seutin V, Engel D (2010) Differences in $\mathrm{Na}^{+}$conductance density and $\mathrm{Na}^{+}$ channel functional properties between dopamine and GABA neurons of the rat substantia nigra. J Neurophysiol 103:3099-3114.

Tepper JM, Damlama M, Trent F (1994) Postnatal changes in the distribution and morphology of rat substantia nigra dopaminergic neurons. Neuroscience 60:469-477.

Thome C, Kelly T, Yanez A, Schultz C, Engelhardt M, Cambridge SB, Both M, Draguhn A, Beck H, Egorov AV (2014) Axon-carrying dendrites convey privileged synaptic input in hippocampal neurons. Neuron 83:14181430.

Tucker KR, Huertas MA, Horn JP, Canavier CC, Levitan ES (2012) Pacemaker rate and depolarization block in nigral dopamine neurons: a somatic sodium channel balancing act. J Neurosci 32:14519-14531.

van Ooyen A, Duijnhouwer J, Remme MW, van Pelt J (2002) The effect of dendritic topology on firing patterns in model neurons. Network $13: 311-325$

Van Wart A, Trimmer JS, Matthews G (2007) Polarized distribution of ion channels within microdomains of the axon initial segment. J Comp Neurol 500:339-352.
Vandecasteele M, Glowinski J, Deniau JM, Venance L (2008) Chemical transmission between dopaminergic neuron pairs. Proc Natl Acad Sci U S A 105:4904-4909.

Vetter P, Roth A, Häusser M (2001) Propagation of action potentials in dendrites depends on dendritic morphology. J Neurophysiol 85:926-937.

Washio H, Takigachi-Hayashi K, Konishi S (1999) Early postnatal development of substantia nigra neurons in rat midbrain slices: hyperpolarization-activated inward current and dopamine-activated current. Neurosci Res 34:91-101.

Weaver CM, Wearne SL (2008) Neuronal firing sensitivity to morphologic and active membrane parameters. PLoS Comput Biol 4:e11.

Wefelmeyer W, Cattaert D, Burrone J (2015) Activity-dependent mismatch between axo-axonic synapses and the axon initial segment controls neuronal output. Proc Natl Acad Sci U S A 112:9757-9762.

Wilson CJ, Callaway JC (2000) Coupled oscillator model of the dopaminergic neuron of the substantia nigra. J Neurophysiol 83:3084-3100.

Yee AG, Forbes B, Cheung PY, Martini A, Burrell MH, Freestone PS, Lipski J (2019) Action potential and calcium dependence of tonic somatodendritic dopamine release in the Substantia Nigra pars compacta. J Neurochem 148:462-479.

Zhou D, Lambert S, Malen PL, Carpenter S, Boland LM, Bennett V (1998) AnkyrinG is required for clustering of voltage-gated $\mathrm{Na}$ channels at axon initial segments and for normal action potential firing. J Cell Biol 143: 1295-1304. 